\title{
Blind spots for neutralino dark matter in the NMSSM
}

\section{Marcin Badziak, Marek Olechowski and Pawet Szczerbiak}

Institute of Theoretical Physics, Faculty of Physics, University of Warsaw, ul. Pasteura 5, PL-02-093 Warsaw, Poland

E-mail: mbadziak@fuw.edu.pl, Marek.0lechowski@fuw.edu.pl,

Pawel.Szczerbiak@fuw.edu.pl

ABSTRACT: Spin-independent cross-section for neutralino dark matter scattering off nuclei is investigated in the NMSSM. Several classes of blind spots for direct detection of singlinoHiggsino dark matter are analytically identified, including such that have no analog in the MSSM. It is shown that mixing of the Higgs doublets with the scalar singlet has a big impact on the position of blind spots in the parameter space. In particular, this mixing allows for more freedom in the sign assignment for the parameters entering the neutralino mass matrix, required for a blind spot to occur, as compared to the MSSM or the NMSSM with decoupled singlet. Moreover, blind spots may occur for any composition of a singlino-Higgsino LSP. Particular attention is paid to cases with the singlet-dominated scalar lighter than the $125 \mathrm{GeV}$ Higgs for which a vanishing tree-level spin-independent scattering cross-section may result from destructive interference between the Higgs and the singlet-dominated scalar exchange. Correlations of the spin-independent scattering cross-section with the Higgs observables are also discussed.

KEYWORDS: Supersymmetry Phenomenology

ARXIV EPRINT: 1512.02472 


\section{Contents}

1 Introduction 1

2 Higgs and neutralino sector of the NMSSM 4

3 Spin-independent scattering cross-section $\quad 6$

$\begin{array}{lll}3.1 & \text { Blind spot conditions } & 7\end{array}$

4 Blind spots without interference effects $\quad 8$

$\begin{array}{llr}4.1 & \text { Without scalar mixing } & 9\end{array}$

4.2 With scalar mixing, $m_{s} \gg m_{h} \quad 12$

$\begin{array}{lll}4.2 .1 & \text { Purity limits } & 12\end{array}$

4.2.2 General Higgsino-singlino LSP 13

5 Blind spots with interference effects between $h$ and $H$ exchange $\quad 18$

$\begin{array}{ll}5.1 \text { Without mixing with singlet } & 18\end{array}$

5.2 Mixing with singlet, $m_{s} \gg m_{h} \quad 20$

$6 \quad$ Blind spots with interference effects between $h$ and $s$ exchange $\quad 22$

$\begin{array}{lll}6.1 & \text { Large } \tan \beta \text { region } & 26\end{array}$

$\begin{array}{lll}7 & \text { Summary } & 27\end{array}$

$\begin{array}{ll}\text { A Useful formulae } & 29\end{array}$

\section{Introduction}

After the recent discovery of the Higgs boson [1,2], probably the most wanted new particle is the one responsible for the observed dark matter (DM) in the Universe. Among extensions of the Standard Model (SM) that provide a candidate for a dark matter particle, supersymmetric models are most attractive. One of the main reasons that kept particle physics community interested in supersymmetric models for more than three decades is their ability to solve the hierarchy problem of the SM. Moreover, in the simplest supersymmetric extensions of the SM the lightest supersymmetric particle (LSP) is stable and generically neutral making it a good dark matter candidate. In most of the supersymmetry breaking schemes the LSP is a neutralino.

One of the most promising ways to search for neutralino dark matter is through its direct interactions with nuclei. In the last couple of years sensitivity of direct dark matter detection experiments improved by several orders of magnitude. The best constraints for the spin-independent (SI) neutralino-nucleon scattering cross-section (for the DM masses 
above $6 \mathrm{GeV}$ ) are provided now by the LUX experiment [3]. In consequence, significant portions of the neutralino sector parameter space has been excluded by LUX. The constraints will become soon even stronger with the forthcoming experiments such as XEXON1T [4] and LZ [5]. Nevertheless, there are points in the parameter space, so-called blind spots, for which the neutralino LSP spin-independent scattering cross-section (almost) vanishes at the tree level. In the vicinity of such blind spots the neutralino LSP is not only consistent with the LUX constraints but, due to the irreducible neutrino background [6], might be never detected in direct detection experiments sensitive only to the SI scattering crosssection. When comparing with the results of DM detection experiments we assume that the considered particle is the main component of DM with the relic density obtained by the Planck satellite [7] (otherwise the experimental bounds on the cross-sections should be re-scaled by the ratio $\left.\Omega_{\text {observed }} / \Omega_{\mathrm{LSP}}\right)$.

Conditions for the existence of blind spots have been already identified in the Minimal Supersymmetric Standard Model (MSSM). In ref. [8] the conditions for MSSM parameters leading to a vanishing Higgs-neutralino-neutralino coupling were found in the limit of decoupled heavy Higgs doublet. Additional blind spots in the MSSM originating from destructive interference between contributions to the scattering amplitude mediated by the $125 \mathrm{GeV}$ Higgs and the heavy Higgs doublet were found in ref. [9]. However, the measured Higgs scalar mass strongly motivates extensions of the MSSM because the $125 \mathrm{GeV}$ Higgs implies in the MSSM relatively heavy stops threatening naturalness of supersymmetry. Substantially lighter stops than in the MSSM can be consistent with the $125 \mathrm{GeV}$ Higgs in the Next-to-Minimal Supersymmetric Standard Model (NMSSM) [10] which is the MSSM supplemented by a gauge singlet chiral superfield. The neutralino sector of the NMSSM is richer than that of the MSSM because it contains, in addition, the fermionic component of the singlet superfield - the singlino. In some part of the parameter space the LSP has a non-negligible singlino component and can be a good dark matter candidate [11-13] but with different properties than those of the LSP in the MSSM. There have been many studies of neutralino dark matter in the NMSSM including predictions for its direct detection, see e.g. refs. [14-20] and references therein. ${ }^{1}$ However, conditions for blind spots in the NMSSM have not been discussed in the literature so far.

The main aim of this paper is to investigate conditions for SI scattering cross-section blind spots for a singlino-Higgsino LSP in the NMSSM. We find a general formula for the blind spot condition and study it in the most interesting and phenomenologically relevant limiting cases, focusing both on small and large $\tan \beta$ regions. First of all, we identify blind spots analogous to those for a gaugino-Higgsino LSP in the MSSM originating from a vanishing Higgs-neutralino-neutralino coupling [8]. Such blind spots were also found in a general singlet-doublet DM model which mimics NMSSM with a Higgsino-singlino DM with a decoupled scalar singlet and heavy MSSM-like doublet [22] (see also ref. [23] for a recent analysis). However, in our analysis we include also the effects of mixing among scalars. We find that inclusion of the mixing with the singlet introduces qualitatively new features to the conditions for blind spots, e.g. allowing certain signs of some parameters that would be

\footnotetext{
${ }^{1}$ Prospects for indirect detection of Higgsino-singlino LSP have also been studied [21].
} 
forbidden if such mixing is neglected. Secondly, we find blind spots analogous to those in the MSSM with the effect of the heavy doublet taken into account [9] and generalize them to the case with the Higgs-singlet mixing included.

Finally, we investigate in great detail the region of the NMSSM parameter space with the singlet-dominated scalar lighter than $125 \mathrm{GeV}$, which is entirely new with respect to the MSSM. This region is particularly interesting because the Higgs-singlet mixing can increase the Higgs boson mass by up to about $6 \mathrm{GeV}$ [24]. While this enhancement of the Higgs mass by mixing effects can be present both for small and large $\tan \beta$, it is worth emphasizing that for large (or moderate) values of $\tan \beta$ this is a unique way to have lighter stops than in the MSSM. Moreover, for large $\tan \beta$ the singlet-dominated scalar coupling to bottom quarks can be strongly suppressed relaxing the LEP constraints on scalars and allowing a substantial correction to the Higgs mass from mixing for a wide range of singlet masses between about 60 and $110 \mathrm{GeV}$ [24] (for small $\tan \beta$ a sizable correction from mixing is allowed only for the singlet mass in the vicinity of the LEP excess at $98 \mathrm{GeV}[25,26])$.

In the case of a light singlet-dominated scalar with sizable mixing with the Higgs scalar, the SI scattering cross-section is generically large, even for not too large values of $\lambda$. The main reason for this is that such a singlet-dominated scalar also mediates the SI scattering cross-section and the corresponding amplitude may even dominate over the one with the SM-like Higgs boson exchange due to the enhancement by a small mass of the singlet-dominated scalar. This phenomenon was identified long before the Higgs scalar discovery $[14,15]$. Recently, points in the parameter space of the NMSSM with strongly suppressed SI direct detection cross-section, consistent with LUX constraints and in some cases even below the irreducible neutrino background for direct detection experiments, were found using sophisticated numerical scans of semi-constrained NMSSM [27]. However, in ref. [27] no explanation was given why such points exist and what are the conditions for the NMSSM parameters required for this suppression to occur. In the present paper we provide analytic understanding for the existence of blind spots in the NMSSM with light singletdominated scalar and a Higgsino-singlino LSP. Such blind spots follow from a destructive interference between the singlet and Higgs exchange in the scattering amplitude. We also discuss the influence of a strongly suppressed coupling of the singlet-dominated scalar to $b$ quarks which is important at large $\tan \beta$. In particular, we find that the presence of a light singlet-dominated scalar gives much more freedom in the LSP composition and, especially for a singlino-dominated LSP, in sign assignments of various NMSSM parameters required for obtaining a blind spot.

The rest of the paper is organized as follows. In section 2 we review some features of the Higgs and neutralino sector of the NMSSM that are important for the analysis of blind spots. In section 3 SI scattering cross-section in the NMSSM is reviewed and general formulae for neutralino blind spots are derived. In the remaining sections blind spot conditions are analyzed in detail in several physically interesting cases and approximations. In section 4 only SM-like Higgs scalar exchange is taken into account. In section 5 the interference effects between two doublet-dominated scalars are analyzed, while section 6 is focused on the case with a light singlet-dominated scalar in which interference effects between such light scalar and the SM-like Higgs scalar become important. Our main findings are summarized in section 7 . 


\section{Higgs and neutralino sector of the NMSSM}

Several versions of NMSSM has been proposed so far [10]. We would like to keep our discussion as general as possible so we assume that the NMSSM specific part of the superpotential and the soft terms have the following general forms:

$$
\begin{aligned}
W_{\mathrm{NMSSM}}= & \left(\mu_{H_{u} H_{d}}+\lambda S\right) H_{u} H_{d}+f(S) \\
-\mathcal{L}_{\text {soft }} \supset & m_{H_{u}}^{2}\left|H_{u}\right|^{2}+m_{H_{d}}^{2}\left|H_{d}\right|^{2}+m_{S}^{2}|S|^{2} \\
& +\left(A_{\lambda} \lambda H_{u} H_{d} S+\frac{1}{3} A_{\kappa} \kappa S^{3}+m_{3}^{2} H_{u} H_{d}+\frac{1}{2} m_{S}^{\prime 2} S^{2}+\xi_{S} S+\text { h.c. }\right)
\end{aligned}
$$

where $S$ is an additional SM-singlet superfield. The first term in (2.1) is the source of the effective Higgsino mass parameter, $\mu_{\mathrm{eff}} \equiv \mu_{H_{u} H_{d}}+\lambda v_{s}$ (we drop the subscript "eff" in the rest of the paper). Using the shift symmetry of $S$ we can put $\mu_{H_{u} H_{d}}=0$. In the simplest version, known as the scale-invariant NMSSM, $m_{3}^{2}=m_{S}^{\prime 2}=\xi_{S}=0$ while $f(S) \equiv \kappa S^{3} / 3$. In more general models $f(S) \equiv \xi_{F} S+\mu^{\prime} S^{2} / 2+\kappa S^{3} / 3$.

There are three neutral CP-even scalar fields, $H_{u}, H_{d}, S$ which are the real parts of excitations around the real vevs, $v_{u} \equiv v \sin \beta, v_{d} \equiv v \cos \beta, v_{s}$ with $v^{2}=v_{u}^{2}+v_{d}^{2} \approx$ $(174 \mathrm{GeV})^{2}$, of the neutral components of the doublets $H_{u}, H_{d}$ and the singlet $S$ (we use the same notation for the doublets and the singlet as for the real parts of their neutral components). It is more convenient for us to work in the basis $(\hat{h}, \hat{H}, \hat{s})$, where $\hat{h}=$ $H_{d} \cos \beta+H_{u} \sin \beta, \hat{H}=H_{d} \sin \beta-H_{u} \cos \beta$ and $\hat{s}=S$. The $\hat{h}$ field has exactly the same couplings to the gauge bosons and fermions as the SM Higgs field. In this basis the scalar mass squared matrix reads:

$$
M^{2}=\left(\begin{array}{ccc}
M_{\hat{h} \hat{h}}^{2} & M_{\hat{h} \hat{H}}^{2} & M_{\hat{h} \hat{s}}^{2} \\
M_{\hat{h} \hat{H}}^{2} & M_{\hat{H} \hat{H}}^{2} & M_{\hat{H} \hat{s}}^{2} \\
M_{\hat{h} \hat{s}}^{2} & M_{\hat{H} \hat{s}}^{2} & M_{\hat{s} \hat{s}}^{2}
\end{array}\right),
$$

where

$$
\begin{aligned}
M_{\hat{h} \hat{h}}^{2} & =M_{Z}^{2} \cos ^{2}(2 \beta)+\lambda^{2} v^{2} \sin ^{2}(2 \beta)+\left(\delta m_{h}^{2}\right)^{\mathrm{rad}} \\
M_{\hat{H} \hat{H}}^{2} & =\left(M_{Z}^{2}-\lambda^{2} v^{2}\right) \sin ^{2}(2 \beta)+\frac{2}{\sin (2 \beta)}\left(\mu A_{\lambda}+\frac{\mu\left\langle\partial_{S} f\right\rangle}{v_{s}}+m_{3}^{2}\right) \\
M_{\hat{s} \hat{s}}^{2} & =\frac{1}{2} \lambda v^{2} \sin 2 \beta\left(\frac{\Lambda}{v_{s}}-\left\langle\partial_{S}^{3} f\right\rangle\right)+\left\langle\left(\partial_{S}^{2} f\right)^{2}+\partial_{S} f \partial_{S}^{3} f\right\rangle-\frac{\left\langle\partial_{S} f \partial_{S}^{2} f\right\rangle}{v_{s}}+A_{\kappa} \kappa v_{s}-\frac{\xi_{S}}{v_{s}}, \\
M_{\hat{h} \hat{H}}^{2} & =\frac{1}{2}\left(M_{Z}^{2}-\lambda^{2} v^{2}\right) \sin 4 \beta \\
M_{\hat{h} \hat{s}}^{2} & =\lambda v(2 \mu-\Lambda \sin 2 \beta), \\
M_{\hat{H} \hat{s}}^{2} & =\lambda v \Lambda \cos 2 \beta
\end{aligned}
$$

and $\Lambda \equiv A_{\lambda}+\left\langle\partial_{S}^{2} f\right\rangle$. We neglected all the radiative corrections except those to $M_{\hat{h} \hat{h}}^{2}$ which we parametrize by $\left(\delta m_{h}^{2}\right)^{\mathrm{rad}}$. The mass eigenstates of $M^{2}$, denoted by $h_{i}\left(\right.$ with $\left.h_{i}=h, H, s\right)$, 
are expressed in terms of the hatted fields with the help of the diagonalization matrix $\tilde{S}:^{2}$

$$
h_{i}=\tilde{S}_{h_{i} \hat{h}} \hat{h}+\tilde{S}_{h_{i} \hat{H}} \hat{H}+\tilde{S}_{h_{i} \hat{s}} \hat{s} .
$$

We will refer to the eigenvalue $h$ as the Higgs scalar and identify it with the $125 \mathrm{GeV}$ scalar discovered by the LHC experiments.

The neutralino mass matrix in NMSSM is 5-dimensional. However, in this work we assume that gauginos are heavy and thus we focus on the sub-matrix describing the three lightest neutralinos:

$$
M_{\chi^{0}}=\left(\begin{array}{ccc}
0 & -\mu & -\lambda v \sin \beta \\
-\mu & 0 & -\lambda v \cos \beta \\
-\lambda v \sin \beta & -\lambda v \cos \beta & \left\langle\partial_{S}^{2} f\right\rangle
\end{array}\right) .
$$

Trading the model dependent term $\left\langle\partial_{S}^{2} f\right\rangle$ for one of the eigenvalues, $m_{\chi_{j}}$, of the above neutralino mass matrix we find the following (exact at the tree level) relations for the neutralino diagonalization matrix elements: ${ }^{3}$

$$
\begin{aligned}
& \frac{N_{j 3}}{N_{j 5}}=\frac{\lambda v}{\mu} \frac{\left(m_{\chi_{j}} / \mu\right) \sin \beta-\cos \beta}{1-\left(m_{\chi_{j}} / \mu\right)^{2}}, \\
& \frac{N_{j 4}}{N_{j 5}}=\frac{\lambda v}{\mu} \frac{\left(m_{\chi_{j}} / \mu\right) \cos \beta-\sin \beta}{1-\left(m_{\chi_{j}} / \mu\right)^{2}},
\end{aligned}
$$

where $j=1,2,3$ and $\left|m_{\chi_{1}}\right| \leq\left|m_{\chi_{2}}\right| \leq\left|m_{\chi_{3}}\right|$. Later we will be interested mainly in the LSP corresponding to $j=1$, so to simplify the notation we will use $m_{\chi} \equiv m_{\chi_{1}}$. Notice that the physical (positive) LSP mass equals to $m_{\mathrm{LSP}} \equiv\left|m_{\chi}\right|$. The sign of $m_{\chi}$ is the same as that of the diagonal singlino entry $\left\langle\partial_{S}^{2} f\right\rangle$ in the neutralino mass matrix (2.11). For $\left|\left\langle\partial_{S}^{2} f\right\rangle\right|<|\mu|$ this is obvious. For bigger values of $\left|\left\langle\partial_{S}^{2} f\right\rangle\right|$ it is also true. In this case the two lightest neutralinos are Higgsino-dominated corresponding to the mass eigenstates close to $\mu$ and $-\mu$. The lighter of them is the one which mixes more strongly with the singlino, and generally the mixing is stronger between states with the diagonal terms of the same sign (unless the corresponding off-diagonal term is exceptionally small).

Using eqs. (2.12) and (2.13) and the fact that the gauginos are decoupled, we can express the ratio of the Higgsino to the singlino components of the LSP as the following function of the LSP mass and the ratio $(\lambda v) / \mu$ :

$$
\frac{1-N_{15}^{2}}{N_{15}^{2}}=\left(\frac{\lambda v}{\mu}\right)^{2} \frac{1+\left(m_{\chi} / \mu\right)^{2}-2\left(m_{\chi} / \mu\right) \sin 2 \beta}{\left[1-\left(m_{\chi} / \mu\right)^{2}\right]^{2}} .
$$

\footnotetext{
${ }^{2}$ The matrix $\tilde{S}$ is related to the commonly used Higgs mixing matrix $S$ by a rotation by the angle $\beta$ in the 2-dimensional space of the weak doublets.

${ }^{3}$ We consider only the $3 \times 3$ sub-matrix (2.11) but we keep the notation from the full $5 \times 5$ neutralino mass matrix i.e. $N_{j 3}, N_{j 4}$ and $N_{j 5}$ denote, respectively, the two Higgsino and the singlino components of the $j$-th neutralino mass eigenstate. The mathematical structure of this matrix is very similar to $3 \times 3$ sub-matrix mixing higgsino with one of the gauginos in the MSSM. Many useful formulae that follow from this matrix can be found in the appendix of ref. [28] with obvious substitutions of the MSSM parameters into the NMSSM ones sitting in the corresponding entries of the $3 \times 3$ sub-matrix.
} 
In our discussion we will consider only positive values of $\lambda$. The results for negative $\lambda$ are exactly the same due to the invariance under the transformation $\lambda \rightarrow-\lambda, \kappa \rightarrow-\kappa$, $\xi_{S} \rightarrow-\xi_{S}, \xi_{F} \rightarrow-\xi_{F}, S \rightarrow-S$ with other fields and couplings unchanged.

\section{Spin-independent scattering cross-section}

The spin-independent cross-section for the LSP interacting with the nucleus with the atomic number $Z$ and the mass number $A$ is given by

$$
\sigma_{\mathrm{SI}}=\frac{4 \mu_{\mathrm{red}}^{2}}{\pi} \frac{\left[Z f^{(p)}+(A-Z) f^{(n)}\right]^{2}}{A^{2}}
$$

where $\mu_{\text {red }}^{2}$ is the reduced mass of the nucleus and the LSP. Usually, the experimental limits concern the cross section $\sigma_{\mathrm{SI}}$ defined as the arithmetic mean of $\sigma_{\mathrm{SI}}^{(p)}$ and $\sigma_{\mathrm{SI}}^{(n)}$. Thus, in the rest of the paper we will follow this convention. When the squarks are heavy the effective couplings $f^{(N)}(N=p, n)$ are dominated by the t-channel exchange of the CP-even scalars [29]:

$$
f^{(N)} \approx \sum_{i=1}^{3} f_{h_{i}}^{(N)} \equiv \sum_{i=1}^{3} \frac{\alpha_{h_{i} \chi \chi} \alpha_{h_{i} N N}}{2 m_{h_{i}}^{2}} .
$$

The couplings of the $i$-th scalar to the LSP and to the nucleon are given, respectively, by

$$
\begin{aligned}
\alpha_{h_{i} \chi \chi}= & \sqrt{2} \lambda\left(S_{i 1} N_{14} N_{15}+S_{i 2} N_{13} N_{15}+S_{i 3} N_{13} N_{14}\right)-\sqrt{2} \kappa S_{i 3} N_{15}^{2} \\
& +g_{1}\left(S_{i 1} N_{11} N_{13}-S_{i 2} N_{11} N_{14}\right)-g_{2}\left(S_{i 1} N_{12} N_{13}-S_{i 2} N_{12} N_{14}\right)
\end{aligned}
$$

and

$$
\alpha_{h_{i} N N}=\frac{m_{N}}{\sqrt{2} v}\left(\frac{S_{i 1}}{\cos \beta} F_{d}^{(N)}+\frac{S_{i 2}}{\sin \beta} F_{u}^{(N)}\right)
$$

In the last equation we introduced the combinations $F_{d}^{(N)}=f_{d}^{(N)}+f_{s}^{(N)}+\frac{2}{27} f_{G}^{(N)}$ and $F_{u}^{(N)}=f_{u}^{(N)}+\frac{4}{27} f_{G}^{(N)}$ of the form factors $f_{q}^{(N)}=m_{N}^{-1}\left\langle N\left|m_{q} q \bar{q}\right| N\right\rangle$ (for $\left.q=u, d, s\right)$ and $f_{G}^{(N)}=1-\sum_{q=u, d, s} f_{q}^{(N)}$. There is still some inconsistency in the literature regarding the values of these form factors. In our numerical calculations we will take them to be: $f_{u}^{(p)}=0.0153, f_{d}^{(p)}=0.0191, f_{s}^{(p)}=0.048, f_{G}^{(p)}=0.921, f_{u}^{(n)}=0.0107, f_{d}^{(n)}=0.0273$, $f_{s}^{(n)}=0.0447, f_{G}^{(n)}=0.917$, which gives the following values of $F^{\prime}$ 's: $F_{u}^{(p)} \approx 0.152, F_{d}^{(p)} \approx$ $0.132, F_{u}^{(n)} \approx 0.147, F_{d}^{(n)} \approx 0.140[32]$.

The couplings of the scalar particles in eqs. (3.3) and (3.4) are expressed in terms of the diagonalization matrices for the scalars and neutralinos ( $S$ and $N$, respectively) written in the usual weak bases. However, for our purposes it will be more convenient to use the scalar diagonalization matrix $\tilde{S}$ defined in $(2.10)$ for the rotated basis $(\hat{h}, \hat{H}, \hat{s})$. Moreover, we are interested in the situation when the LSP is Higgsino-singlino like with negligible contributions from gauginos i.e. $N_{11} \approx 0 \approx N_{12}$. Then, the expressions (3.3) and (3.4) are approximated by:

$$
\begin{gathered}
\alpha_{h_{i} \chi \chi} \approx \sqrt{2} \lambda\left[\tilde{S}_{h_{i} \hat{h}} N_{15}\left(N_{13} \sin \beta+N_{14} \cos \beta\right)+\tilde{S}_{h_{i} \hat{H}} N_{15}\left(N_{14} \sin \beta-N_{13} \cos \beta\right)\right. \\
\left.+\tilde{S}_{h_{i} \hat{s}}\left(N_{13} N_{14}-\frac{\kappa}{\lambda} N_{15}^{2}\right)\right],
\end{gathered}
$$




$$
\alpha_{h_{i} N N} \approx \frac{m_{N}}{\sqrt{2} v}\left[\tilde{S}_{h_{i} \hat{h}}\left(F_{d}^{(N)}+F_{u}^{(N)}\right)+\tilde{S}_{h_{i} \hat{H}}\left(\tan \beta F_{d}^{(N)}-\frac{1}{\tan \beta} F_{u}^{(N)}\right)\right] .
$$

The formulae for the spin-independent cross-section in a general case are rather complicated so in order to make some expressions more compact it is useful to define the following parameters:

$$
\mathcal{A}_{h_{i}} \equiv \frac{\tilde{S}_{h_{i} \hat{h}}\left(F_{d}^{(N)}+F_{u}^{(N)}\right)+\tilde{S}_{h_{i} \hat{H}}\left(\tan \beta F_{d}^{(N)}-\cot \beta F_{u}^{(N)}\right)}{\tilde{S}_{h \hat{h}}\left(F_{d}^{(N)}+F_{u}^{(N)}\right)+\tilde{S}_{h \hat{H}}\left(\tan \beta F_{d}^{(N)}-\cot \beta F_{u}^{(N)}\right)} \frac{\tilde{h}_{i} \hat{h}_{i}}{\tilde{S}_{h \hat{h}}}\left(\frac{m_{h}}{m_{h_{i}}}\right)^{2} .
$$

This is the product of the coupling to a nucleon, the propagator and the value of the leading component for the scalar $h_{i}$ divided by the same product for $h$. Of course, $\mathcal{A}_{h}=1$ and $\mathcal{A}_{H}$ $\left(\mathcal{A}_{s}\right)$ vanishes in the limit $m_{H} \rightarrow \infty\left(m_{s} \rightarrow \infty\right)$. We define also some combinations of the above parameters:

$$
\mathcal{B}_{\hat{h}_{i}} \equiv \frac{\tilde{S}_{h \hat{h}_{i}}}{\tilde{S}_{h \hat{h}}}+\mathcal{A}_{H} \frac{\tilde{S}_{H \hat{h}_{i}}}{\tilde{S}_{H \hat{H}}}+\mathcal{A}_{s} \frac{\tilde{S}_{s \hat{h}_{i}}}{\tilde{S}_{s \hat{s}}}
$$

which encode the information on the scalar sector (mixing, masses and couplings to the nucleons). Using the above definitions we rewrite (3.2) in the form

$$
\begin{aligned}
& f^{(N)} \approx \frac{\lambda}{\sqrt{2}} \frac{\alpha_{h N N}}{m_{h}^{2}} \tilde{S}_{h \hat{h}}\left\{\mathcal{B}_{\hat{h}} N_{15}\left(N_{13} \sin \beta+N_{14} \cos \beta\right)\right. \\
& \left.+\mathcal{B}_{\hat{H}} N_{15}\left(N_{14} \sin \beta-N_{13} \cos \beta\right)+\mathcal{B}_{\hat{s}}\left(N_{13} N_{14}-\frac{\kappa}{\lambda} N_{15}^{2}\right)\right\} .
\end{aligned}
$$

\subsection{Blind spot conditions}

The blind spots are defined as those points in the parameter space for which the LSPnucleon cross-section vanishes. From eq. (3.9) we obtain the following general blind spot condition

$\mathcal{B}_{\hat{h}} N_{15}\left(N_{13} \sin \beta+N_{14} \cos \beta\right)+\mathcal{B}_{\hat{H}} N_{15}\left(N_{14} \sin \beta-N_{13} \cos \beta\right)+\mathcal{B}_{\hat{s}}\left(N_{13} N_{14}-\frac{\kappa}{\lambda} N_{15}^{2}\right)=0$.

This condition simplifies very much for the case of a pure Higgsino $\left(N_{15}=0\right)$ or a pure singlino $\left(N_{13}=N_{14}=0\right)$ LSP. For such pure states the blind spot condition reads

$$
\mathcal{B}_{\hat{s}}=0 \text {. }
$$

For a mixed Higgsino-singlino LSP it is convenient to introduce the parameter

$$
\eta \equiv \frac{N_{15}\left(N_{13} \sin \beta+N_{14} \cos \beta\right)}{N_{13} N_{14}-\frac{\kappa}{\lambda} N_{15}^{2}}
$$

which is totally described by the neutralino sector and the dimensionless couplings of the singlet superfield in the superpotential i.e. $\lambda$ and $\kappa \cdot{ }^{4}$ This parameter vanishes for neutralinos which are pure (Higgsino or singlino) states. Its absolute value grows with

\footnotetext{
${ }^{4}$ Note that in $\mathbb{Z}_{3}$-NMSSM $\kappa$ controls also the neutralino mass parameter.
} 
the increasing admixture of the sub-dominant components and has a maximum (or even a pole) for a specific highly mixed composition. The position and height of such maximum depend on the parameters of the model. Whether there is a pole or a maximum depends on the relative signs of some parameters. The details are given in the appendix.

The parameter $\eta$ can be used to rewrite eq. (3.10) as

$$
\left(\mathcal{B}_{\hat{h}}+\eta^{-1} \mathcal{B}_{\hat{s}}\right) N_{15}\left(N_{13} \sin \beta+N_{14} \cos \beta\right)+\mathcal{B}_{\hat{H}} N_{15}\left(N_{14} \sin \beta-N_{13} \cos \beta\right)=0 .
$$

After using eqs. (2.12) and (2.13), the above general blind spot condition may be cast in the form

$$
\left(\mathcal{B}_{\hat{h}}+\eta^{-1} \mathcal{B}_{\hat{s}}\right)\left(\frac{m_{\chi}}{\mu}-\sin 2 \beta\right)+\mathcal{B}_{\hat{H}} \cos 2 \beta=0 .
$$

For a highly Higgsino-dominated LSP, for which $N_{15}$ and $\eta$ have very small values, it is better to rewrite eq. (3.10) as:

$$
\left(\eta \mathcal{B}_{\hat{h}}+\mathcal{B}_{\hat{s}}\right)\left(N_{13} N_{14}-\frac{\kappa}{\lambda} N_{15}^{2}\right)+\mathcal{B}_{\hat{H}} N_{15}\left(N_{14} \sin \beta-N_{13} \cos \beta\right)=0 .
$$

After applying eqs. (2.12) and (2.13), this blind spot condition for a highly Higgsinodominated LSP takes the form

$$
\left(\eta \mathcal{B}_{\hat{h}}+\mathcal{B}_{\hat{s}}\right)\left[\left(1+\left(\frac{m_{\chi}}{\mu}\right)^{2}\right) \frac{\sin 2 \beta}{2}-\frac{m_{\chi}}{\mu}-\frac{\kappa}{\lambda}\left(\frac{1-\left(\frac{m_{\chi}}{\mu}\right)^{2}}{\frac{\lambda v}{\mu}}\right)^{2}\right]+\mathcal{B}_{\hat{H}} \frac{1-\left(\frac{m_{\chi}}{\mu}\right)^{2}}{\frac{\lambda v}{\mu}} \cos 2 \beta=0 .
$$

In many cases considered in this paper the contribution from $\mathcal{B}_{\hat{H}}$ may be neglected. Then the blind spot conditions simplifies to

$$
\frac{\mathcal{B}_{\hat{s}}}{\mathcal{B}_{\hat{h}}}=-\eta
$$

In the rest of the paper we will analyze in some detail the above blind spot conditions for several cases and approximations.

\section{Blind spots without interference effects}

Let us start with a case in which $f_{s}^{(N)}$ and $f_{H}^{(N)}$ are negligible so blind spots correspond to $f_{h}^{(N)} \approx 0$ and result from an accidentally vanishing $h \chi \chi$ coupling. ${ }^{5}$ Generically the contributions from $s$ and $H$ exchange are very small when these scalars are very heavy. Then, the quantities $\mathcal{A}_{H}$ and $\mathcal{A}_{s}$ defined in (3.7) are negligible and eq. (3.8) reduces to $\mathcal{B}_{\hat{h}_{i}}=\tilde{S}_{h \hat{h}_{i}} / \tilde{S}_{h \hat{h}}$. The situation is qualitatively different depending on whether the Higgs scalar mixes with other scalars or not so we discuss these cases separately in the following subsections.

\footnotetext{
${ }^{5}$ We do not consider in this paper the possibility that a blind spot may originate from vanishing coupling of the Higgs scalar to nucleons, i.e. vanishing $\alpha_{h N N}$ in eq. (3.9). In principle, this may happen if $h$ has a non-zero $\hat{H}$ component and $\tan \beta$ is large enough so that the second term in the square bracket of eq. (3.6) for $h_{i}=h$ cancels the first (usually dominant) term in that bracket.
} 


\subsection{Without scalar mixing}

Without mixing with (heavy) $\hat{H}$ and $\hat{s}$, the lightest scalar $h$ has the same couplings as the SM Higgs. In our notation this corresponds to $\mathcal{B}_{\hat{h}}=1, \mathcal{B}_{\hat{H}}=\mathcal{B}_{\hat{s}}=0$. The condition (3.11) is fulfilled so the SI scattering cross-section vanishes when the LSP is a pure singlino or pure Higgsino state. For a general Higgsino-singlino LSP the amplitude (3.9) results in the following approximate formula for this cross-section:

$$
\sigma_{\mathrm{SI}} \approx k \cdot 10^{-45} \mathrm{~cm}^{2}\left(\frac{\lambda}{0.1}\right)^{2} \frac{N_{15}^{2}\left(1-N_{15}^{2}\right)}{(0.5)^{2}}
$$

where $k$ depends on the value of $\tan \beta$ and typically is of order $\mathcal{O}(1)$. This implies that a highly mixed Higgsino-singlino LSP is strongly constrained by the LUX results unless $\lambda$ is very small. For $\lambda$ which is not small, these constraints may be avoided if there is some (partial) cancellation between the two terms in the bracket multiplying $\mathcal{B}_{\hat{h}}$ in eq. (3.9) (which results in an unusually small value of $k$ in (4.1)). Such cancellation is equivalent to vanishing of the parameter $\eta$ (defined in (3.12)) and leads, according to eq. (3.17), to a blind spot. Therefore, highly mixed Higgsino-singlino neutralino dark matter with not very small $\lambda$ may be viable only in very special parts of the parameter space, close to such blind spots. The blind spot condition (3.14) for the present values of the $\mathcal{B}_{\hat{h}_{i}}$ parameters, $\mathcal{B}_{\hat{h}}=1, \mathcal{B}_{\hat{H}}=\mathcal{B}_{\hat{s}}=0$, simplifies to:

$$
\frac{m_{\chi}}{\mu}-\sin 2 \beta=0
$$

This result is analogous to the one obtained in [8] for the Higgsino-gaugino LSP in MSSM, but with opposite sign between the two terms in the 1.h.s. This difference stems from the fact that both off-diagonal terms, mixing the singlino with two Higgsinos, have the same sign while the two analogous terms, mixing any of the gauginos with the Higgsinos, have opposite signs. Notice that if $\tan \beta$ is not small, the blind spot condition implies a singlinodominated LSP $\left(\left|m_{\chi}\right| \ll|\mu|\right)$ for which $f_{h}^{(N)}$ is suppressed anyway. Thus, for a Higgsinosinglino LSP and large $\tan \beta$ this kind of a blind spot does not help much in suppression of SI scattering cross-section. On the other hand, for small $\tan \beta$ and highly mixed singlinoHiggsino LSP the blind spot condition may be satisfied provided that $\mu\left\langle\partial_{S}^{2} f\right\rangle$ is positive. ${ }^{6}$ This is illustrated in figure 1 where the SI scattering cross-section is plotted as a function of the diagonal singlino mass term $\left\langle\partial_{S}^{2} f\right\rangle$ (equal to $2 \kappa v_{s}$ in the scale-invariant NMSSM) for $\lambda=0.6$, for two values of $\tan \beta$ and for both signs of $\mu$. It can be seen that for small values of $\tan \beta$ ( $=2$ in our example) the cross-section is substantially above the LUX limit $^{7}$ for $\mu\left\langle\partial_{S}^{2} f\right\rangle<0$. As expected, the largest cross-section is for $\left\langle\partial_{S}^{2} f\right\rangle \approx-\mu$ corresponding to the maximal singlino-Higgsino mixing. Even in the region with $\left\langle\partial_{S}^{2} f\right\rangle$ several times larger

\footnotetext{
${ }^{6}$ As we explained in section 2 , the sign of $m_{\chi}$ is the same as that of the diagonal singlino entry, $\left\langle\partial_{S}^{2} f\right\rangle$, in the neutralino mass matrix. In the scale-invariant NMSSM and in our convention with $\lambda>0$, the sign of the product $\mu\left\langle\partial_{S}^{2} f\right\rangle$ is the same as the sign of $\kappa$.

${ }^{7}$ We assume in this work that the relic density of DM is equal to the value consistent with the results obtained by the Planck satellite [7]. If it would not be the case i.e. if the relic density would be smaller in a specific scenario, the experimental bounds should be appropriately rescaled (and hence relaxed).
} 

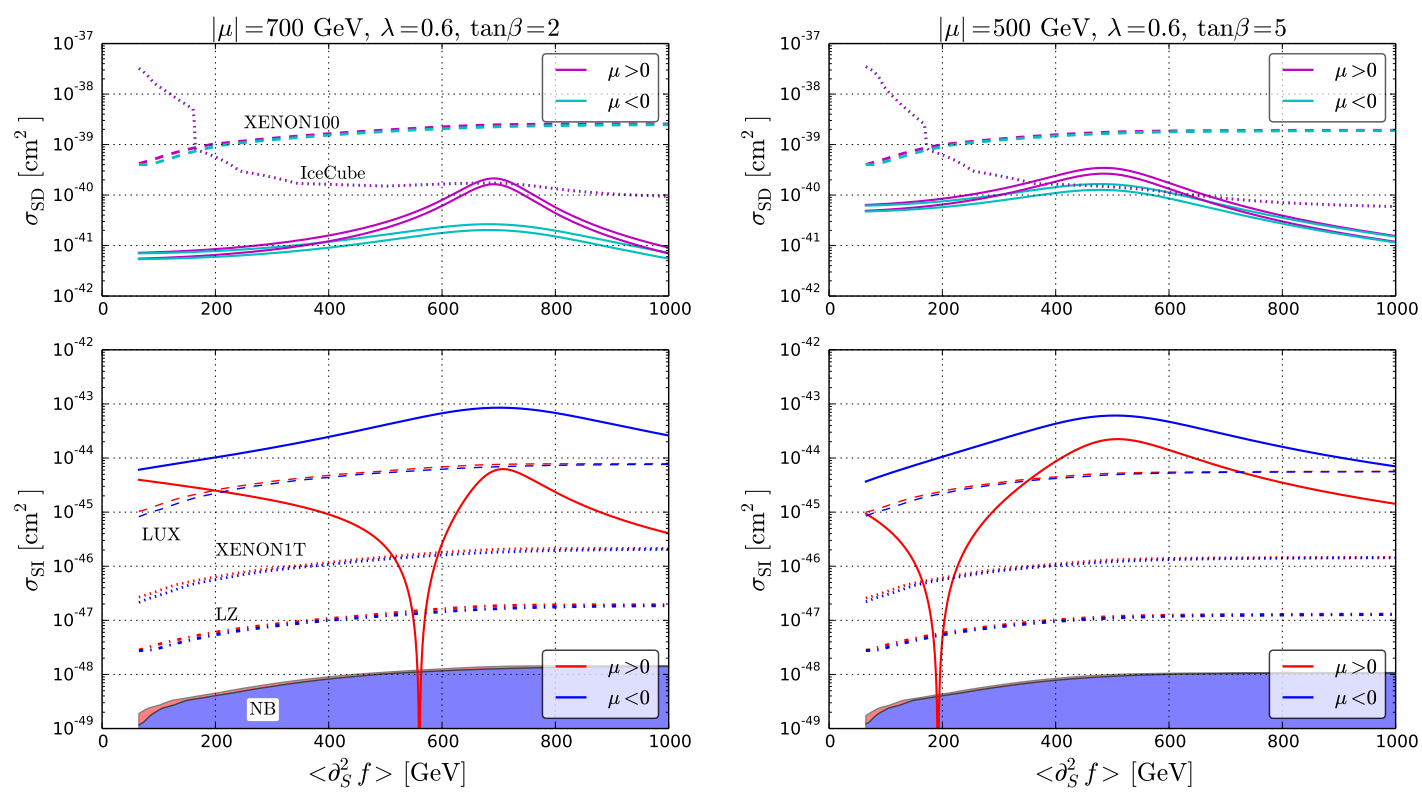

Figure 1. Lower panels: the solid lines show the LSP spin-independent cross-section as a function of the diagonal singlino mass term $\left\langle\partial_{S}^{2} f\right\rangle$ for positive (red) and negative (blue) values of parameter $\mu$. The dashed, dotted and dashed-dotted lines indicate the corresponding upper bounds from, respectively, LUX [3], XENON1T [4] and LZ [5] experiments. The colored areas at the bottom depict the neutrino background (NB) regions [6]. Upper panels: the solid lines show the LSP spindependent cross-section on neutrons (lower) and protons (upper) for positive (purple) and negative (cyan) values of parameter $\mu$. The dashed and dotted lines denote the corresponding upper limits from, respectively, XENON100 [33] and IceCube [34] (see details in text). For all used experimental bounds we assume that the relic density of the LSP is equal to the observed value [7] (otherwise these bounds should be re-scaled by the ratio $\left.\Omega_{\text {observed }} / \Omega_{\mathrm{LSP}}\right)$.

than $|\mu|$, i.e. for a Higgsino-dominated LSP, a small singlino component is enough to push the cross-section above the LUX limit. The cross-section is below the LUX upper bound only for the LSP with a very tiny Higgsino admixture i.e. for very large values of $\left\langle\partial_{S}^{2} f\right\rangle$.

The situation is drastically different for $\mu\left\langle\partial_{S}^{2} f\right\rangle>0$. The cross-section is substantially smaller in this case and the LUX limit is satisfied for a wide range of values of $\left\langle\partial_{S}^{2} f\right\rangle$. One can see that most of this region is within the reach of the XENON1T experiment. However, in the vicinity of the blind spot defined by the condition (4.2) (corresponding to $m_{\chi}=0.8 \mu$ for $\tan \beta=2$ ) none of the future SI direct detection experiments will be able to exclude (or discover) such a singlino-Higgsino LSP. On the other hand, this region may be probed with DM detection experiments sensitive to SD interactions. The most stringent model independent upper bound on SD cross-section is provided by XENON100 for neutrons [33]. The limits on the SD DM-proton cross-section, provided by the indirect detection experiment IceCube [34], depend strongly on assumed dominant annihilation channels of dark matter particles. Generically in NMSSM with small $\tan \beta$ and decoupled scalars the singlino-dominated LSP annihilates mainly into $t \bar{t}$ (if the LSP mass is above the top quark mass) while the Higgsino-dominated LSP annihilates mainly into $W W$ and $Z Z$ 
(if kinematically allowed). The IceCube limits for DM annihilating dominantly to $W W$, $Z Z$ or $t \bar{t}$ are stronger than the XENON100 limits (on SD DM-neutron cross-section) for dark matter masses above about $100 \mathrm{GeV}$ [34]. In the upper panels of figure 1 SD crosssections are shown with superimposed XENON100 and IceCube limits. The IceCube limits are computed assuming the LSP annihilation channels as obtained from MicrOMEGAs [32] with the spectrum computed by NMSSMTools $4.8 .2[30,31]$ for the model parameters as in figure 1 and $\kappa=A_{\kappa}=m_{S}^{\prime 2}=\xi_{F}=0$ as well as $A_{\lambda}, \xi_{S}$ and $m_{3}^{2}$ chosen in such way that $\tilde{S}_{h \hat{s}} \approx 0, m_{a_{1}}, m_{s}, m_{H} \approx 3 \mathrm{TeV}$. The SD cross-sections we calculated using eqs. (A.8)(A.10) (which, as we checked, give results in very good agreement with those obtained with the help of MicrOMEGAs). Note that for $\tan \beta=2, \lambda=0.6$ and $|\mu|=700 \mathrm{GeV}$ in the vicinity of the SI cross-section blind spot the SD cross-section is not much below the current IceCube limit. Since the SD cross-section is larger for larger Higgsino-singlino mixing, which is proportional to $(\lambda v / \mu)$, the SI blind spot is harder to probe by testing the $\mathrm{SD}$ cross-section if $\lambda$ is smaller and/or $|\mu|$ is bigger (see eq. (A.10)). Moreover, for larger $\tan \beta$ the SI blind spot occurs for smaller values of $\left|m_{\chi} / \mu\right|$, for which the SD cross-section is smaller (because the LSP is more singlino-dominated). Thus, for larger $\tan \beta$ smaller values of $|\mu|$ are consistent with the IceCube limits, as can be seen from the upper right panel of figure 1 . We should note also that if LSPs annihilate mainly to $b \bar{b}$, which may happen e.g. when there is a light sbottom in the spectrum, the IceCube limits are always weaker than the XENON100 ones. In such a case the SI blind spots are much harder to probe via SD detection experiments, though not impossible.

We should also comment on the fact that for $\tan \beta=1$ and $m_{\chi} \mu>0$ the blind spot condition (4.2) is always satisfied as long as $|\mu|<\left\langle\partial_{S}^{2} f\right\rangle$ because in such a case the LSP has a vanishing singlino component so $m_{\chi}=\mu$. Value of $\tan \beta=1$ is relevant in the context of $\lambda$ SUSY $[35,36]$ and will be particularly hard to probe because in such situation also SD scattering cross-section vanishes, see eqs. (A.8)-(A.9).

The properties of the LSP change with the increasing value of $\tan \beta$. The difference between values of $\sigma_{\mathrm{SI}}$ for two signs of $\mu$ decreases. As a result, already for $\tan \beta=5$, a substantial part of the parameter space with positive $\mu$ and $\left\langle\partial_{S}^{2} f\right\rangle>|\mu|$ is excluded by the LUX data. At the same time, the SI cross-section for negative $\mu$ decreases and goes below the LUX upper bound for the LSP with the Higgsino admixture bigger (i.e. for smaller values of $\left.\left\langle\partial_{S}^{2} f\right\rangle\right)$ than in the case of smaller $\tan \beta$. What does not change is that there is a blind spot only for positive $\mu$. The position of the blind spot moves towards smaller $\left\langle\partial_{S}^{2} f\right\rangle$ corresponding to a more singlino-dominated LSP.

As mentioned before, in our analysis we use the tree-level approximation for the SI cross-sections. Inclusion of loop corrections does not affect our main conclusion that for $m_{\chi} \mu>0$ a blind spot for the SI cross-section exists. The loop effects may only change slightly the position of a given blind spot. The computation of even dominant loop corrections to the SI cross-section is quite involved. The results are known only for neutralinos which are pure interaction eigenstates [37-39]. For a pure Higgsino LSP the radiatively corrected SI cross-section is of order $\mathcal{O}\left(10^{-49}\right) \mathrm{cm}^{2}$ so below the irreducible neutrino background. One should, however, note that such a small SI cross-section is a consequence of quite strong cancellations between contributions from several different (gluon and quark, 
including twist-2) operators, some of which contribute as much as $\mathcal{O}\left(10^{-47}\right) \mathrm{cm}^{2}$. Computation of the loop corrected SI cross-section for (highly) mixed Higgsino-singlino LSP is beyond the scope of this work. We conservatively estimate that in such a case the loop correction to the tree-level cross-section does not exceed a few times $10^{-48} \mathrm{~cm}^{2}$ i.e. the biggest twist-2 operator contribution for a pure Higgsino with appropriately reduced couplings to the EW gauge bosons. Loop corrections of this size would result in a small shift of the position of a blind spot: by less than one per cent in terms of $\left\langle\partial_{S}^{2} f\right\rangle$. We checked (using MicrOMEGAs/NMSSMTools) that similar size of a shift of a blind spot position occurs when the gauginos are not completely decoupled but have masses of order $2 \mathrm{TeV}$. One should stress that the approximations used in our analysis result only in some small uncertainties of the exact positions of the blind spots but do not influence their existence.

\subsection{With scalar mixing, $m_{s} \gg m_{h}$}

Next we consider the situation when the contributions to $\sigma_{\mathrm{SI}}$ from the exchange of $H$ and $s$ may still be neglected $\left(\mathcal{A}_{H}=\mathcal{A}_{s}=0\right)$ but the mixing of $h$ with other scalars may play some role because now $\mathcal{B}_{\hat{h}}=1, \mathcal{B}_{\hat{H}}=\tilde{S}_{h \hat{H}} / \tilde{S}_{h \hat{h}}, \mathcal{B}_{\hat{s}}=\tilde{S}_{h \hat{s}} / \tilde{S}_{h \hat{h}}$. The effective LSP-nucleon coupling is obtained by putting these expressions for the $\mathcal{B}_{\hat{h}_{i}}$ parameters into eq. (3.9). The fact that $\mathcal{B}_{\hat{H}}$ and $\mathcal{B}_{\hat{s}}$ do not vanish implies that in the present case a blind spot may exist for $\eta \neq 0$. However, as we shall see the blind condition still requires $\eta$ to be very small. In the rest of this subsection we discuss the blind spot conditions in some interesting limits.

\subsubsection{Purity limits}

Before analyzing the general mixed LSP let us discuss limiting cases of a pure Higgsino and a pure singlino for which the effective coupling to a nucleon (3.9) simplifies to:

$$
f_{h}^{(N)} \approx \frac{\alpha_{h N N}}{\sqrt{2} m_{h}^{2}} \tilde{S}_{h \hat{h}} \mathcal{B}_{\hat{s}} C=\frac{\alpha_{h N N}}{\sqrt{2} m_{h}^{2}} C \tilde{S}_{h \hat{s}}
$$

where $C$ is equal to $\lambda N_{13} N_{14}\left(-\kappa N_{15}^{2}\right)$ for the pure Higgsino (singlino). Note that, in contrast to MSSM where the effective tree-level coupling of the pure Higgsino to a nucleon vanishes [8], the effective coupling in NMSSM does not vanish as long as the singlet scalar mixes with the Higgs doublet i.e. when $\tilde{S}_{h \hat{s}} \neq 0$. Similarly, such non-zero singlet-Higgs mixing implies a non-vanishing SI scattering cross-section also for a pure singlino. Notice that the magnitude of the effective coupling of the LSP to nucleons, hence also the SI scattering cross-section, is controlled by $\kappa$ for the singlino and by $\lambda$ for the Higgsino.

In order to get a feeling about typical (i.e. without significant cancellations in the amplitude) magnitudes of the SI scattering cross-section it is enlightening to show simplified formulae assuming that the $\hat{H}$ component of the SM-like Higgs mass eigenstate is negligible: ${ }^{8}$

$$
\sigma_{\mathrm{SI}} \approx \mathcal{O}(0.25) \cdot 10^{-45} \mathrm{~cm}^{2}\left(\frac{\lambda}{0.1}\right)^{2}\left(\frac{\tilde{S}_{h \hat{s}}^{2} \tilde{S}_{h \hat{h}}^{2}}{0.1 \cdot 0.9}\right)
$$

\footnotetext{
${ }^{8}$ This approximation is justified since the $\hat{H}$ component modifies the Higgs scalar coupling to the bottom quark which is very constrained by the LHC data (because modifications of this coupling strongly affect the Higgs scalar total decay width and, in consequence, all of the Higgs scalar branching ratios).
} 
for a Higgsino LSP,

$$
\sigma_{\mathrm{SI}} \approx \mathcal{O}(1) \cdot 10^{-45} \mathrm{~cm}^{2}\left(\frac{\kappa}{0.1}\right)^{2}\left(\frac{\tilde{S}_{h \hat{s}}^{2} \tilde{S}_{h \hat{h}}^{2}}{0.1 \cdot 0.9}\right)
$$

for a singlino LSP. It is clear from the above formulae that, unless the couplings and/or the singlet-Higgs mixing are very small, pure Higgsino and singlino neutralino dark matter is generically either excluded by LUX or is within the reach of the forthcoming direct detection experiments such as XENON1T (so it can be soon found or excluded). In particular, for widely considered small $\tan \beta$ and $\lambda \sim 0.6$ the SI scattering cross-section for the Higgsino LSP is typically of order $10^{-44} \mathrm{~cm}^{2}$, which is above the LUX limit for a wide range of its masses.

\subsubsection{General Higgsino-singlino LSP}

For the LSP which is a general Higgsino-singlino mixture there are several non-zero contributions to $f_{h}^{(N)}$ including the one proportional to $\mathcal{B}_{\hat{h}}$ (see eq. (3.9)) which on its own leads to SI scattering cross-section of order $10^{-45} \mathrm{~cm}^{2}$ for $\lambda \approx 0.1$, as discussed in subsection 4.1. Thus, if those contributions add constructively in the amplitude the resulting cross-section is even bigger. On the other hand, if those contributions add destructively a new kind of a blind spot may appear.

In the present case the blind spot condition (3.10) can be rewritten in the form (3.14) as:

$$
\frac{m_{\chi}}{\mu}-\sin 2 \beta=-\frac{\tilde{S}_{h \hat{H}}}{\tilde{S}_{h \hat{h}}} \cos 2 \beta-\frac{\tilde{S}_{h \hat{s}}}{\tilde{S}_{h \hat{h}}} \eta^{-1}\left(\frac{m_{\chi}}{\mu}-\sin 2 \beta\right),
$$

with $\eta$ given by eq. (A.2). Notice that the term in the bracket cancels with the same term present in the numerator of (A.2). The r.h.s. of the above expression quantifies the correction to eq. (4.2), coming from the mixing among scalars. It is tempting to check whether adding this correction can change the conclusion of subsection 4.1. The first term, proportional to $\tilde{S}_{h \hat{H}}$, is typically very small since $\tilde{S}_{h \hat{H}}$ is strongly constrained by the LHC measurements of the $h b \bar{b}$ coupling. This corresponds to $\mathcal{B}_{\hat{H}} \approx 0$. Thus, it cannot change qualitatively the conclusions of the case without scalar mixing. The situation differs greatly in the case of the second term on the r.h.s. of eq. (4.6) which may give important corrections to the simple blind spot condition (4.2).

For the discussion of the corrections to the blind spot condition it is useful to express $\tilde{S}_{h \hat{s}}$ in terms of the NMSSM parameters (for $m_{s} \gg m_{h}$ assumed in this section):

$$
\frac{\tilde{S}_{h \hat{s}}}{\tilde{S}_{h \hat{h}}} \approx \lambda v \frac{(\Lambda \sin 2 \beta-2 \mu)}{m_{s}^{2}} \approx \operatorname{sgn}(\Lambda \sin 2 \beta-2 \mu) \frac{\sqrt{2\left|\Delta_{\text {mix }}\right| m_{h}}}{m_{s}} .
$$

In the last approximate equality we introduced $\Delta_{\text {mix }}$, defined as

$$
\Delta_{\text {mix }} \equiv m_{h}-\hat{M}_{h h},
$$

which parameterizes the correction to the Higgs scalar mass due to its mixing with the remaining scalars, mainly with the singlet $\hat{s}$. For $m_{s}>m_{h}$ this correction is always negative 
so its magnitude is desired to be small. Notice that smallness of $\left|\Delta_{\text {mix }}\right|$ usually requires some cancellation between the two terms in the bracket (especially for large $\lambda$ ) in the middle part of formula (4.7) which implies $\mu \Lambda>0$. Notice also that the requirement of small $\left|\Delta_{\text {mix }}\right|$, say smaller than $\mathcal{O}(1) \mathrm{GeV}$, implies $\left(\tilde{S}_{h \hat{s}} / \tilde{S}_{h \hat{h}}\right) \lesssim 0.1\left(m_{h} / m_{s}\right)$. Therefore, in order to have a strong modification of the blind spot condition, at least one of the other factors in the second term of the r.h.s. of eq. (4.6) must be much larger than one. This sets the condition for the NMSSM parameter space which depends on the composition of the LSP.

Because in the rest of this subsection we will neglect the term proportional to $\tilde{S}_{h \hat{H}}$ in (4.6) our blind spot conditions will be of the form (3.17):

$$
\frac{\tilde{S}_{h \hat{s}}}{\tilde{S}_{h \hat{h}}} \approx-\eta .
$$

One can see that for small $\hat{h}-\hat{s}$ mixing we demand also small $|\eta|$. The dependence of $\eta$ on the LSP composition and mass is explicit in eq. (A.2). Parameter $\eta$ may be small either because the numerator in (A.2) is small or because the denominator is large. The first possibility corresponds to the standard blind spot (4.2). The second possibility requires (at least) one of the terms in the denominator to be large. In the case of a highly mixed $\operatorname{LSP}\left(1-N_{15}^{2}\right) / N_{15}=\mathcal{O}(1)$ and the denominator may be large only when $|\kappa| \gg|\lambda|$. This, however, is limited by the perturbativity conditions. Moreover, both sides of eq. (4.9) must have the same sign which, using (4.7) and (A.2), gives the condition

$$
\left.\operatorname{sgn}\left(\kappa\left(m_{\chi}-\mu \sin 2 \beta\right)\right)=-\operatorname{sgn}(\eta)=\operatorname{sgn}\left(\tilde{S}_{h \hat{s}}\right)=\operatorname{sgn}(\Lambda \sin 2 \beta-2 \mu)\right) .
$$

It follows that for $m_{\chi} \mu<0$ a blind spot is possible only when the combination of the parameters $\kappa\left(\frac{\Lambda}{\mu} \sin 2 \beta-2\right)$ is also negative. In addition, $|\eta|$ is smaller (i.e. better for a blind spot with small $\left|\Delta_{\text {mix }}\right|$ ) when both terms in the denominator of eq. (A.2) are of the same sign which is the case when

$$
\operatorname{sgn}(\kappa)=-\operatorname{sgn}\left(\left(1+\left(\frac{m_{\chi}}{\mu}\right)^{2}\right) \sin 2 \beta-2 \frac{m_{\chi}}{\mu}\right) .
$$

In the present case with a small value of $\tilde{S}_{h \hat{s}}$ it is easier to have a blind spot when the LSP is strongly dominated by the singlino (or Higgsino) component because then either $N_{15}^{2} /\left(1-N_{15}^{2}\right)$ or $\left(1-N_{15}^{2}\right) / N_{15}^{2}$ in the numerator of (A.2) is large. Let us now discuss these two situations.

Singlino-dominated LSP. It has been already noted that for pure singlino $\eta$ is exactly zero. However, a pure singlino can be obtained only for infinite value of $|\mu|$. Very large $|\mu|$ is undesirable for multiple reasons, including naturalness arguments. For natural values of $|\mu|$ even if the LSP is singlino-dominated some Higgsino component is always present which may have non-negligible contribution to $\eta$, hence also to a blind spot condition. Notice also that for a given value of $\mu$ a minimal value of the Higgsino component of the LSP grows with $\lambda$ since the latter controls the magnitude of the singlino-Higgsino mixing. In what follows we study the impact of a non-zero Higgsino component for the existence of a blind spot. 
The blind spot condition (3.17) with $\eta$ given by eq. (A.3) takes the following form

$$
\frac{m_{\chi}}{\mu}-\sin 2 \beta \approx \operatorname{sgn}\left(\frac{\Lambda}{\mu} \sin 2 \beta-2\right) \frac{\kappa}{\lambda} \sqrt{\frac{\left|\Delta_{\text {mix }}\right| m_{h}}{m_{s}^{2}} \frac{2 N_{15}^{2}}{1-N_{15}^{2}}\left(1+\left(\frac{m_{\chi}}{\mu}\right)^{2}-2 \frac{m_{\chi}}{\mu} \sin 2 \beta\right)} .
$$

For a strongly singlino-dominated LSP its mass $\left|m_{\chi}\right|$ is much smaller than $|\mu|$ so the first term in the l.h.s. of the above equation is rather small and the blind spot condition without the scalar mixing effects (i.e. with the r.h.s. neglected) can be fulfilled only for appropriately large $\tan \beta$ and for positive $m_{\chi} \mu$. Now we will check whether the scalar mixing effects may lead to blind spots with smaller values of $\tan \beta$ and/or negative $m_{\chi} \mu$. Such changes are possible only when the r.h.s of (4.12) is negative because decreasing of $\tan \beta$ and changing the sign of $m_{\chi} \mu$ both give negative corrections to the l.h.s of the above blind spot condition. This gives the condition $\left(\frac{\Lambda}{\mu} \sin 2 \beta-2\right) \kappa<0$. In addition, the absolute value of the r.h.s of (4.12) should not be very small in order to give a substantial modification of the blind spot condition. The biggest such value is necessary when one wants simultaneously to decrease $\tan \beta$ and have negative $m_{\chi} \mu$. Let us now discuss such an extreme modification of blind spots.

In the region of large $\lambda \sim 0.6$ and small $\tan \beta \sim 2$, the l.h.s. of (4.12) is $\mathcal{O}(1)$ while the r.h.s. is generically very small. The reason is that, in addition to the suppression by small $\left|\Delta_{\text {mix }}\right|$, the r.h.s. is suppressed also by the factor $\kappa / \lambda$ because for $\lambda \sim 0.6$ the perturbativity up to the GUT scale requires $\kappa \lesssim 0.4$ [10]. The only way to enhance the r.h.s. would be by the factor $1 / \sqrt{1-N_{15}^{2}}$. However, the r.h.s. could be of order $\mathcal{O}(1)$ only for extremely pure singlino corresponding to $|\mu| \gg \lambda v$. For large $\lambda$ this translates to extremely large, hence very unnatural, values of $|\mu|$. For example, for $|\kappa|=0.1,\left|\Delta_{\text {mix }}\right|=1 \mathrm{GeV}$ and $m_{s}=500 \mathrm{GeV},|\mu|$ would have to be $\mathcal{O}(20) \mathrm{TeV}$. Thus, we conclude that for large $\lambda$ and small $\tan \beta$ it is not possible to have a blind spot for a singlino-dominated LSP with $m_{\chi} \mu<0$, unless the Higgsino is extremely heavy. For $m_{\chi} \mu>0$ such a blind spot can occur only if the standard blind spot condition (4.2) is approximately satisfied. This can be seen in figure 2 (in all plots presented in this paper the LEP and LHC Higgs constraints (at $2 \sigma$ level) are satisfied unless otherwise stated).

The situation changes if $\lambda$ is small. In such a case the r.h.s. of (4.12) can be enhanced both by $\kappa / \lambda$ and by $1 / \sqrt{1-N_{15}^{2}}$ for not so huge values of $|\mu|$. Then a blind spot may appear for $m_{\chi} \mu<0$ and/or small $\tan \beta$ provided that at least one of these factors is large enough (of course only when $\left(\frac{\Lambda}{\mu} \sin 2 \beta-2\right) \kappa<0$ ). It can be seen from the left panel of figure 2 that for $|\mu|=500 \mathrm{GeV}$ a blind spot with $m_{\chi} \mu<0$ may appear for $\lambda \lesssim 0.2$ without violating perturbativity constraints. For larger values of $|\mu|$ larger values of $\lambda$ may allow for a blind spot due to decreasing of the Higgsino component with increasing $|\mu|$.

We note that it is easier to relax the IceCube constraints on the SD cross-section when $|\kappa|$ is not small. This is because for big values of $|\kappa|$ the LSP annihilates dominantly (via the s-channel exchange of a singlet-like pseudoscalar) into a singlet-like scalar and pseudoscalar (if the latter is light enough and LSP has non-negligible singlino component). We have verified with MicrOMEGAs that for $|\kappa| \sim \mathcal{O}(0.1)$ this is indeed the dominant annihilation channel for a singlino-dominated LSP. The IceCube collaboration [34] does not provide 

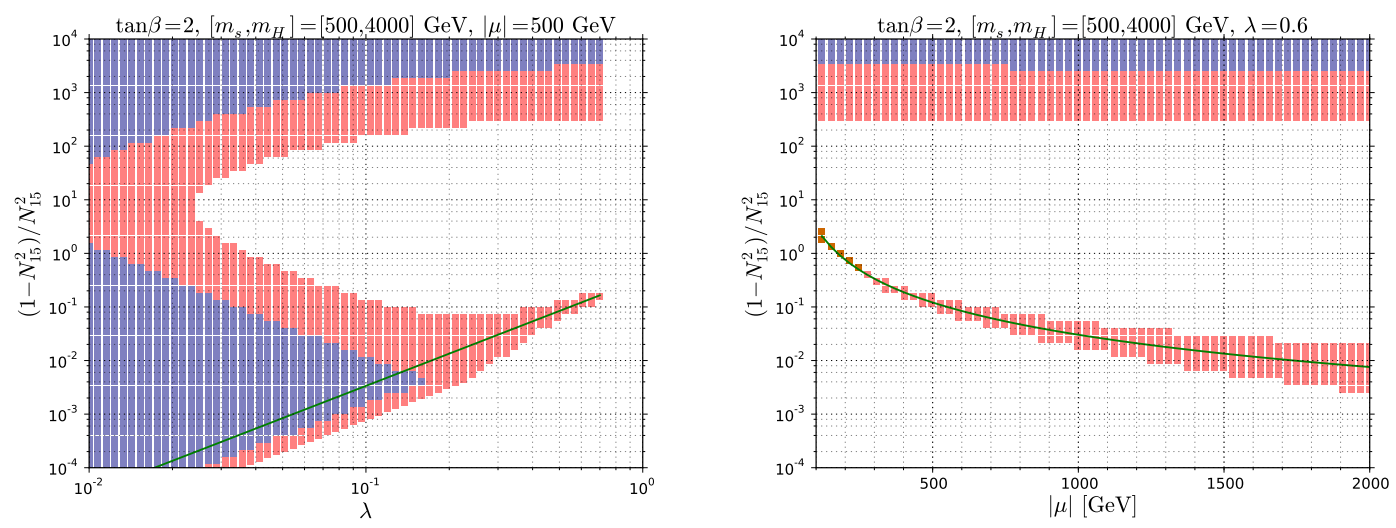

Figure 2. Left: regions of the plane $\left(\lambda,\left(1-N_{15}^{2}\right) / N_{15}^{2}\right)$ with the SI cross-section that can be below the neutrino background for $m_{\chi} \mu>0$ (red) and $m_{\chi} \mu<0$ (blue), while keeping $10^{-3} \leq\left|\Delta_{\text {mix }}\right| \leq$ $1 \mathrm{GeV}$ and $5 \cdot 10^{-3} \leq|\kappa| \leq 0.3$. Right: the same as in the left panel but as a function of $|\mu|$ and fixed $\lambda=0.6$. Green line correspond to the standard blind spot condition (4.2). Brown points on the green line for $|\mu| \approx 120-250 \mathrm{GeV}$ are excluded by the XENON100 constraints on the SD scattering cross-section [33] (see also figure 6). All points are consistent with the LHC Higgs data at $2 \sigma$.
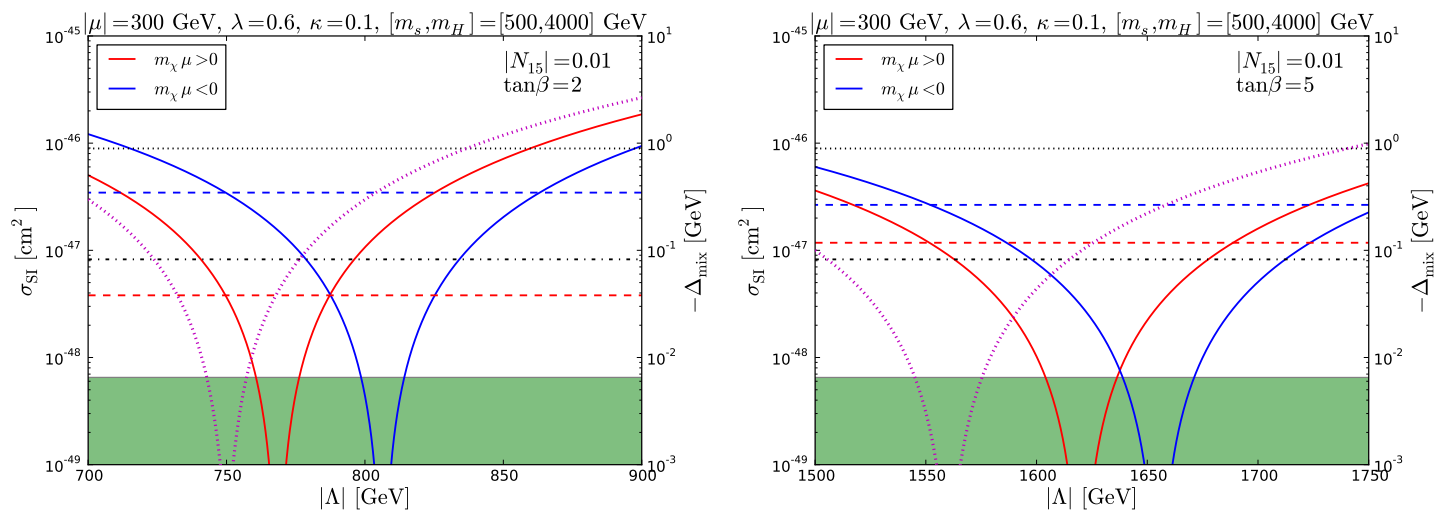

Figure 3. Spin-independent scattering cross-section (solid lines) for a Higgsino-dominated LSP as a function of $|\Lambda|$ which controls the size of $\left|\Delta_{\text {mix }}\right|$ (depicted by coloured dotted curve). Parameters $\lambda,|\mu|$ and $\tan \beta$ are the same as in figure 1. Blue and red dashed horizontal lines correspond to cross-section with mixing between scalars neglected $\left(m_{s}, m_{H} \rightarrow \infty\right)$, whereas black dotted and dashed-dotted lines denote the XENON1T and LZ upper bound, respectively. Green region depicts the neutrino background area. The SD cross-section in the vicinity of blind spots is below the sensitivity of IceCube (independently of the assumed dominant annihilation channel).

limits on the SD cross-section with such an annihilation pattern. It is beyond the scope of the present paper to use the IceCube data to accurately calculate limits for such a case. However, we expect that such limits would be weaker than for DM annihilating into pairs of the SM Higgs bosons because a light singlet-like pseudoscalar decays much more often into the bottom quarks and does not decay into the gauge bosons. Hence, we expect such limit to be comparable to or only slightly better than the one obtained by XENON100. 
Higgsino-dominated LSP. As we discussed in subsection 4.2.1, for a pure Higgsino the SI cross-section is proportional to the $\hat{h}-\hat{s}$ mixing which for $m_{s}>m_{h}$ is preferred to be small to avoid large negative $\Delta_{\text {mix }}$. This implies that for small values of $\left|\Delta_{\text {mix }}\right|$ the LUX constraints on a strongly Higgsino-dominated LSP are generically satisfied. However, this is not the case for future direct detection experiments so the discussion of blind spots is interesting also in this case.

There are no blind spots for a strongly Higgsino-dominated LSP if the contributions from the mixing with $H$ and $s$ scalars are negligible. The reason is that for $m_{\chi} \approx \mu$ the condition (4.2) could be fulfilled only for $\tan \beta$ very close to 1 . Let us check whether this conclusion changes after taking into account the effects of mixing in the scalar sector.

For a Higgsino-dominated neutralino the second term in the denominator in (A.2) may be neglected (unless $\kappa \gg \lambda$ ). Then, substituting (4.7) and $\eta$ given by eq. (A.4) into (3.17), we get the following blind spot condition

$$
\frac{m_{\chi}}{\mu}-\sin 2 \beta \approx-\operatorname{sgn}\left(\frac{\Lambda}{\mu} \sin 2 \beta-2\right) \sqrt{\frac{\left|\Delta_{\text {mix }}\right| m_{h}}{m_{s}^{2}} \frac{1-N_{15}^{2}}{2 N_{15}^{2}}} \frac{\left(1+\left(\frac{m_{\chi}}{\mu}\right)^{2}\right) \sin 2 \beta-2 \frac{m_{\chi}}{\mu}}{\sqrt{1+\left(\frac{m_{\chi}}{\mu}\right)^{2}-2 \frac{m_{\chi}}{\mu} \sin 2 \beta}} .
$$

For a strongly Higgsino-dominated LSP the ratio $\left(m_{\chi} / \mu\right)^{2}$ is very close to 1 so the numerator of the last factor in the r.h.s of the above equation is to a very good precision proportional to the combination $m_{\chi} / \mu-\sin 2 \beta$. So, there are two ways to fulfill the last equation: either both sides vanish or the factor multiplying $m_{\chi} / \mu-\sin 2 \beta$ on the r.h.s is close to 1 . Thus, in the case of a Higgsino-dominated LSP there are two kinds of blind spots. First, like in the case without scalar mixing, is given by condition (4.2) and requires values of $\tan \beta$ very close to 1 and $m_{\chi}$ of the same sign as $\mu$. The second kind of blind spots is given by the condition

$$
1 \approx \operatorname{sgn}\left(\frac{\Lambda}{\mu} \sin 2 \beta-2\right) \frac{\sqrt{\left|\Delta_{\text {mix }}\right| m_{h}}}{\left|N_{15}\right| m_{s}} \frac{1}{\sqrt{1-\operatorname{sgn}\left(m_{\chi} \mu\right) \sin 2 \beta}},
$$

which may be fulfilled only when $(\Lambda / \mu)>(2 / \sin 2 \beta)$.

Notice that for a Higgsino-dominated LSP, i.e. small $\left|N_{15}\right|$, it follows from the last equation that $\left|\Delta_{\text {mix }}\right|$ is preferred to be small for a blind spot to occur. Thus, the tuning of parameters required to keep $\left|\Delta_{\text {mix }}\right|$ small automatically gives some suppression of the SI scattering cross-section, provided that $(\Lambda / \mu)>(2 / \sin 2 \beta)$. However, the strength of this suppression depends on some other parameters. For example, for a fixed value of the singlino component in the LSP, $N_{15}$, it depends on the sign of $\mu$. This follows from the last factor in the r.h.s. of eq. (4.14) and is illustrated in figure 3 for $\lambda=0.6$ and two values of $\tan \beta$. The value of $\left|\Delta_{\text {mix }}\right|$ is bigger when $\mu$ (and in this case also $\Lambda$ ) is negative. As usually, the dependence on the sign of $\mu$ is more pronounced for smaller values of $\tan \beta$. For $\tan \beta=2$ the value of $\left|\Delta_{\text {mix }}\right|$ for negative $\mu$ is about an order of magnitude bigger than for positive $\mu$. So, for a given LSP composition, a blind spot with positive $m_{\chi} \mu$ is preferred because it has a bigger Higgs mass. Indeed, it can be seen in figure 2 that for 
$\left|\Delta_{\text {mix }}\right|<1 \mathrm{GeV}$ and $m_{\chi} \mu>0$ a larger singlino component of the LSP would be allowed if constraints on the SI cross-section would reach the level of the neutrino background than for $m_{\chi} \mu<0$. This fact can be understood from eq. (4.14). Moreover, for a given admixture of the singlino in the LSP larger values of $\lambda$ would be possible for $m_{\chi} \mu>0$.

Let us also point out that for large $\lambda \sim 0.7$, the perturbativity up to the GUT scale requires $\kappa \lesssim 0.3$ which in the scale-invariant NMSSM implies that the diagonal singlino mass term is smaller than $|\mu|$, hence the LSP would be dominated by the singlino. Therefore, the above situation can be realized only in general NMSSM in which the LSP can be Higgsino-dominated provided that $\mu^{\prime}$ parameter (defined below eq. (2.2)) is large enough.

\section{Blind spots with interference effects between $h$ and $H$ exchange}

Let us now consider the case in which $f_{h}^{(N)}$ is not necessarily small but interferes destructively with the contribution $f_{H}^{(N)}$ mediated by the heavy Higgs doublet. This kind of blind spots in the context of MSSM was identified in [9] and can be realized if $H$ is not too heavy and $\tan \beta$ is large. In such a case the coupling of $H$ to down quarks, hence also to nucleons, may be enhanced by large $\tan \beta$ which could compensate the suppression of $f_{H}^{(N)}$ by $m_{H}^{-2}$ resulting in a non-negligible $\mathcal{A}_{H}$ defined in eq. (3.7). In this section we neglect the contribution from the $s$ exchange and set $\mathcal{A}_{s}$ to zero.

\subsection{Without mixing with singlet}

In the case of negligible mixing of the scalar doublets with the scalar singlet the $\mathcal{B}_{\hat{h}_{i}}$ parameters are given by

$$
\mathcal{B}_{\hat{h}} \approx 1+\mathcal{A}_{H} \frac{\tilde{S}_{H \hat{h}}}{\tilde{S}_{H \hat{H}}}, \quad \mathcal{B}_{\hat{H}} \approx \frac{\tilde{S}_{h \hat{H}}}{\tilde{S}_{h \hat{h}}}+\mathcal{A}_{H}, \quad \mathcal{B}_{\hat{s}} \approx 0 .
$$

The mixing between the doublets is small and may be approximated as

$$
\frac{\tilde{S}_{h \hat{H}}}{\tilde{S}_{h \hat{h}}} \approx-\frac{\tilde{S}_{H \hat{h}}}{\tilde{S}_{H \hat{H}}} \approx \frac{2\left(M_{Z}^{2}-\lambda^{2} v^{2}\right)}{m_{H}^{2} \tan \beta} .
$$

The last equality was obtained under two assumptions: we assumed that there is no mixing of the singlet scalar with the doublets ${ }^{9}$ and that $\tan \beta \gg 1$. The former assumption is specific for the present subsection. The latter one is necessary because only then $f_{H}^{(N)}$ contribution to $\sigma_{\mathrm{SI}}$ can compete with $f_{h}^{(N)}$ one. The $\hat{h}-\hat{H}$ mixing given by the last equation is suppressed by large values of $\tan \beta$ and $m_{H}^{2}$. This mixing should be small also from the phenomenological point of view. A non-negligible $\hat{H}$ component in $h$ results for large $\tan \beta$ in strong deviations from the SM predictions of the Higgs scalar branching ratios (because of substantial alteration of the Higgs scalar coupling to bottom quarks) which is constrained by the LHC Higgs measurements.

\footnotetext{
${ }^{9}$ Quite often the contribution to the $\hat{H}$ component of $h$, generated via mixing of both scalar doublets with the singlet scalar, is bigger than the contribution coming directly from the off-diagonal $M_{\hat{h} \hat{H}}^{2}$ entry (2.7) in the Higgs mass matrix (2.3).
} 
When $\mathcal{B}_{\hat{s}}=0$, the blind spot condition (3.14) can be written as

$$
\frac{m_{\chi}}{\mu}-\sin 2 \beta=-\frac{\frac{\tilde{S}_{h \hat{H}}}{\tilde{S}_{h \hat{h}}}+\mathcal{A}_{H}}{1-\mathcal{A}_{H} \frac{\tilde{S}_{h \hat{H}}}{\frac{\tilde{S}_{h \hat{h}}}{2}}} \cos 2 \beta
$$

In the case of large $\tan \beta$ and negligible $\hat{h}-\hat{H}$ mixing, the expression (3.7) for $h_{i}=H$ simplifies to

$$
\mathcal{A}_{H} \approx \frac{\left(\tan \beta F_{d}^{(N)}-\cot \beta F_{u}^{(N)}\right)}{\left(F_{d}^{(N)}+F_{u}^{(N)}\right)}\left(\frac{m_{h}}{m_{H}}\right)^{2} \approx \frac{F_{d}^{(N)}}{F_{d}^{(N)}+F_{u}^{(N)}}\left(\frac{m_{h}}{m_{H}}\right)^{2} \tan \beta \approx\left(\frac{m_{h}}{m_{H}}\right)^{2} \frac{\tan \beta}{2} .
$$

Then the blind spot condition (5.3) takes the form

$$
\frac{m_{\chi}}{\mu}-\sin 2 \beta \approx\left(\frac{m_{h}}{m_{H}}\right)^{2} \frac{\tan \beta}{2} .
$$

This is a similar result to the one obtained in MSSM [9], but for the singlino-Higgsino LSP, rather than the gaugino-Higgsino one. Note, that $\operatorname{sgn}\left(m_{\chi} \mu\right)=1$ is required in contrast to MSSM. It follows from (5.5) that a non-negligible contribution from $\mathcal{A}_{H} \approx\left(m_{h} / m_{H}\right)^{2} \tan \beta$ leads to a bigger Higgsino component of the LSP necessary to obtain a blind spot. However, the LHC experiments have set lower mass limits on the MSSM-like Higgs bosons, which are stronger for larger $\tan \beta$. At large $\tan \beta$, the most stringent constraints on $m_{H}$ come from the ATLAS [40] and CMS [41] searches in the $H / A \rightarrow \tau \tau$ channel. The results of those searches were interpreted in the context of MSSM as constraints on the $m_{A}$-tan $\beta$ plane. These limits can be applied to NMSSM in generic cases and it is typically a good approximation to identify lower limits on $m_{H}$ for a given $\tan \beta$ with the corresponding ones on $m_{A}$. After taking into account these limits one finds generically $\mathcal{A}_{H} \lesssim \mathcal{O}(0.5)$. In the left panel of figure 4 the black line corresponds to a blind spot (5.5) for $\tan \beta=15$ and $m_{H}=500 \mathrm{GeV}$ (resulting in $\mathcal{A}_{H} \approx 0.5$ ) which demonstrates that the Higgsino component of the LSP at a blind spot with large $\tan \beta$ can be increased when effects of the $H$ exchange are not negligible.

We should also comment on the fact that NMSSM provides a framework for relaxing the experimental constraints on $m_{H}$, hence also on $\mathcal{A}_{H}$. Namely, the mass of the MSSMlike pseudoscalar can be very different from $m_{H}$ if one admits mixing of the MSSM-like pseudoscalar with the singlet-dominated pseudoscalar (such mixing can be present even if mixing in the CP-even Higgs sector is strongly suppressed). In such a case, the lower mass limit becomes weaker if the mixing effects push up the MSSM-like pseudoscalar mass substantially above $m_{H}$. While recasting the LHC constraints on such a scenario is beyond the scope of this work, it seems viable that this effect may allow for $H$ light enough to have $\mathcal{A}_{H} \sim \mathcal{O}(1)$. If this is the case, a blind spot at large $\tan \beta$ would exist also for a highly mixed Higgsino-singlino LSP. This would be in contrast to the case with only $h$ exchange for which at large $\tan \beta$ a blind spot cannot exist with $\left|m_{\chi}\right| \approx|\mu|$, see eq. (4.2) and the green line in the left panel of figure 4 . 


\subsection{Mixing with singlet, $m_{s} \gg m_{h}$}

If the mixing with the singlet scalar is taken into account the parameter $\mathcal{B}_{\hat{s}}$ is no longer vanishing. Neglecting the much smaller mixing between the two doublets we get

$$
\mathcal{B}_{\hat{h}} \approx 1, \quad \mathcal{B}_{\hat{H}} \approx \mathcal{A}_{H}, \quad \mathcal{B}_{\hat{s}} \approx \frac{\tilde{S}_{h \hat{s}}}{\tilde{S}_{h \hat{h}}}+\mathcal{A}_{H} \frac{\tilde{S}_{H \hat{s}}}{\tilde{S}_{H \hat{H}}}
$$

and

$$
\mathcal{A}_{H} \approx \frac{2 \tilde{S}_{H \hat{h}}+\tilde{S}_{H \hat{H}} \tan \beta}{2 \tilde{S}_{h \hat{h}}+\tilde{S}_{h \hat{H}} \tan \beta} \frac{\tilde{S}_{H \hat{H}}}{\tilde{S}_{h \hat{h}}}\left(\frac{m_{h}}{m_{H}}\right)^{2},
$$

where we disregarded the difference between $F_{d}^{(N)}$ and $F_{u}^{(N)}$ and assumed $\tan \beta \gg 1$. Adopting these approximations we get the blind spot condition very similar to that given in eq. (4.6), namely:

$$
\frac{m_{\chi}}{\mu}-\sin 2 \beta \simeq-\mathcal{A}_{H} \cos 2 \beta-\left(\frac{\tilde{S}_{h \hat{s}}}{\tilde{S}_{h \hat{h}}}+\mathcal{A}_{H} \frac{\tilde{S}_{H \hat{s}}}{\tilde{S}_{H \hat{H}}}\right) \eta^{-1}\left(\frac{m_{\chi}}{\mu}-\sin 2 \beta\right),
$$

with $\eta$ given by eq. (A.2). The corrections to (4.6), coming from a non-zero amplitude $f_{H}^{(N)}$ and represented by terms proportional to $\mathcal{A}_{H}$, modify both terms in the r.h.s. of (4.6) by shifting the "small" components of the Higgs scalar. Whether one can neglect one of the terms in the first bracket in the above equation, depends not only on the value of $\mathcal{A}_{H}$ but also on the sizes of the $\hat{s}$ components in the scalars $h$ and $H$. The ratio of these two terms can be written as (the dominant components $\tilde{S}_{h \hat{h}}$ and $\tilde{S}_{H \hat{H}}$ are not very different from 1)

$$
\frac{\tilde{S}_{h \hat{s}}}{\mathcal{A}_{H} \tilde{S}_{H \hat{s}}} \operatorname{sgn}(2 \mu-\Lambda \sin 2 \beta)\left(\frac{m_{H}^{2}}{m_{s}^{2}}-1\right) \frac{\sqrt{2\left|\Delta_{\text {mix }}\right| m_{h}}}{\lambda v} \frac{m_{s}}{\Lambda} \frac{m_{H}^{2}}{m_{h}^{2}} \frac{2}{\tan \beta} .
$$

In the following, we focus on the case with $m_{s} \gg m_{H}$, otherwise the assumption of this section that $f_{s}^{(N)}$ is negligible while $f_{H}^{(N)}$ is taken into account would be typically violated. Taking the limit $m_{s} \rightarrow \infty$, while keeping $\Delta_{\text {mix }}$ constant (by adjusting $\Lambda$ and $\mu$ appropriately), the above ratio blows up which means that the contribution proportional to $\mathcal{A}_{H}$ in the second term of the r.h.s. of eq. (5.8) is negligible. Then, the blind spot condition differs from the one (4.6) without $f_{H}^{(N)}$ only by the term $-\mathcal{A}_{H} \cos 2 \beta$ which is always positive and might be $\mathcal{O}(1)$. In consequence, the contribution from $f_{H}^{(N)}$ makes it harder to obtain a blind spot with $m_{\chi} \mu<0$.

Some qualitatively new features may be present only if $m_{s}$ is in the intermediate regime and the ratio (5.9) is small. Note that the factor in eq. (5.9) involving $\left|\Delta_{\text {mix }}\right|$ can be approximately written as $(0.01 / \lambda) \sqrt{\left|\Delta_{\text {mix }}\right| /(1 \mathrm{GeV})}$ so the ratio (5.9) is indeed generically small in the phenomenologically most interesting case of small $\left|\Delta_{\text {mix }}\right|$. The ratio could become large only for very small values of $\lambda$ and/or for $m_{s} \gg \Lambda$. Note, however, that under the assumption of small $\left|\Delta_{\text {mix }}\right|$ and large $\tan \beta$ it follows from eq. (4.7) that $\Lambda \sim \mu \tan \beta$ so the ratio (5.9) is small, unless $m_{s}$ is several orders of magnitude bigger than $|\mu|$. This motivates us to assume in the rest of this section the case of $m_{s} \gg m_{H}$ but with the term 

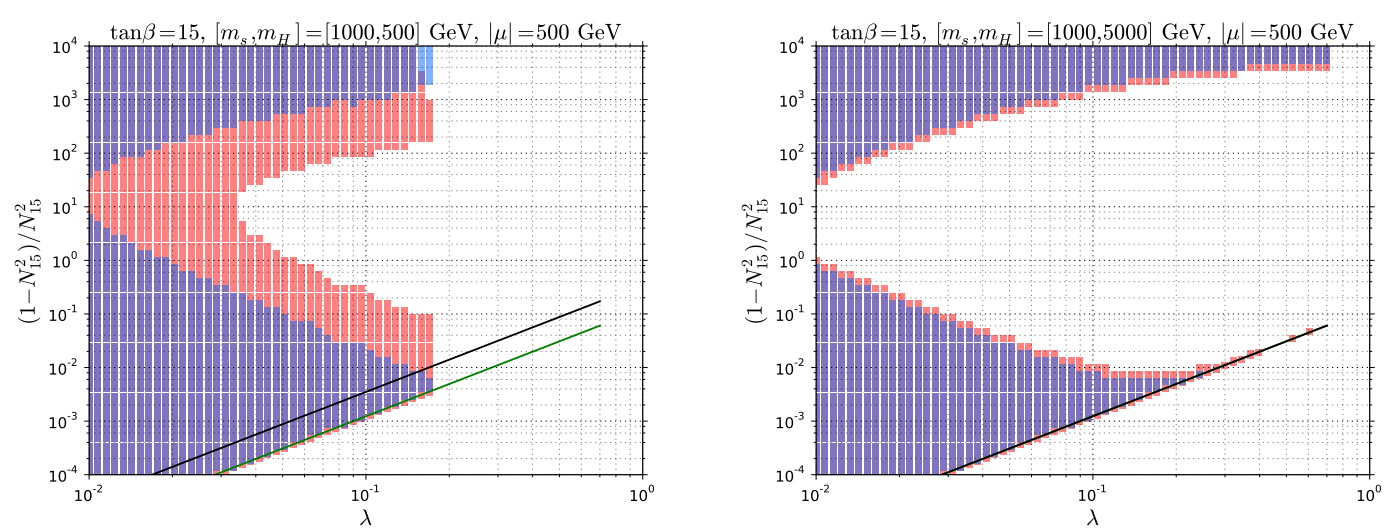

Figure 4. The same as in the left panel of figure 2 but for $\tan \beta=15$ and $m_{H}=500 \mathrm{GeV}$ (left) or $m_{H}=5 \mathrm{TeV}$ (right). Green and black lines correspond to eq. (4.2) and (5.5) respectively. All points are consistent with the LHC Higgs data at $2 \sigma$.

proportional to $\tilde{S}_{h \hat{s}}$ in the r.h.s. of eq. (5.8) neglected. Then, the blind spot condition can be simplified using:

$$
\frac{\tilde{S}_{H \hat{s}}}{\tilde{S}_{H \hat{H}}} \approx \frac{\lambda v \Lambda}{m_{s}^{2}},
$$

which is valid as long as $\lambda v \Lambda$ is small in comparison with $m_{s}^{2}$. From the above equation it should be clear that for large enough $\Lambda$ and $\mathcal{A}_{H} \sim \mathcal{O}(1)$ one can obtain $\left|\mathcal{A}_{H} \frac{\tilde{S}_{H \hat{s}}}{\tilde{S}_{H \hat{H}}}\right| \gg\left|\frac{\tilde{S}_{h \hat{s}}}{\tilde{S}_{h \hat{h}}}\right|$. In such a case the blind spot condition is well approximated by:

$$
\frac{m_{\chi}}{\mu}-\sin 2 \beta \approx \mathcal{A}_{H}\left[1-\frac{\lambda v \Lambda}{m_{s}^{2}} \eta^{-1}\left(\frac{m_{\chi}}{\mu}-\sin 2 \beta\right)\right] .
$$

As already noted in the previous subsection, for $m_{\chi} \mu>0$ it is easier to have a blind spot for a highly mixed Higgsino-singlino LSP. Indeed, it can be seen in figure 4 that at large $\tan \beta$ with light enough $H$ a blind spot is possible for any composition of the LSP. For $m_{\chi} \mu<0$ the situation is different. If the mixing in the scalar sector is small, only the first term in the square bracket in (5.11) is relevant which makes it harder to obtain a blind spot. So in order to have a blind spot with $m_{\chi} \mu<0$ the second term in this bracket must be larger in magnitude. However, this term may be sizable only for small $|\eta|$, i.e. for the LSP which is either dominated by singlino or Higgsino. Therefore, there are no blind spots for a highly mixed Higgsino-singlino LSP with $m_{\chi} \mu<0$. Nevertheless, for large enough $\hat{H}-\hat{s}$ mixing somewhat bigger Higgsino or singlino component may be possible for large $\tan \beta$ if $H$ is light enough, as can be seen from figure 4 .

Notice, however, that for large $\tan \beta$ and relatively light $H$ the value of $\lambda$ exhibits a stronger upper bound. This follows from our requirement that negative $\Delta_{\text {mix }}$ should have rather small absolute value. Indeed, $\left|\Delta_{\text {mix }}\right|$ is small if $\Lambda \approx \mu \tan \beta$ (in order to suppress $\left.M_{\hat{h} \hat{s}}^{2}\right)$ which results in very large, multi-TeV values of $\Lambda$. This in turn implies big $M_{\hat{H} \hat{s}}^{2}$ unless $\lambda$ is small. Nevertheless, the upper bound on $\lambda$ should not be considered problematic since 
there is no strong motivation for big $\lambda$ when $\tan \beta$ is large, which is necessary for this kind of a blind spot.

\section{Blind spots with interference effects between $h$ and $s$ exchange}

Now we turn our attention to a case in which the contributions to the scattering amplitude from the Higgs scalar and the singlet-dominated scalar are comparable. This does not have its analog in MSSM so is particularly interesting. In the presence of non-negligible mixing between the singlet and the Higgs doublet $f_{s}^{(N)}$ is generically large if $m_{s}<m_{h}$. Light singlet-dominated scalar with sizable mixing with the Higgs scalar is particularly well motivated since it can enhance the Higgs scalar mass even by $6 \mathrm{GeV}$ as compared to the MSSM, allowing for relatively light stops in NMSSM, even for large $\tan \beta$ [24].

It was already noticed some time ago $[14,15]$ that the contribution from the singletdominated scalar to the scattering amplitude can be significantly larger than the Higgs contribution. Nowadays, such a possibility is excluded by the current constraints from the direct detection experiments and it is more interesting to study the case in which $f_{s}^{(N)}$ and $f_{h}^{(N)}$ are similar in magnitude and interfere destructively. ${ }^{10}$

We neglect the mixing with the heavy scalar $H$ with one exception - we will keep the terms proportional to $(\tan \beta-\cot \beta) \tilde{S}_{h_{i} \hat{H}}$ in $(3.6)$ for $h_{i}=s, h .{ }^{11}$ This approximation leads to the following relations

$$
\frac{\tilde{S}_{s \hat{s}}}{\tilde{S}_{h \hat{h}}} \approx 1, \quad \gamma \equiv \frac{\tilde{S}_{h \hat{s}}}{\tilde{S}_{h \hat{h}}} \approx-\frac{\tilde{S}_{h \hat{s}}}{\tilde{S}_{s \hat{s}}}
$$

In the last equation we introduced parameter $\gamma$ which may be related to $\Delta_{\text {mix }}$ by the following equation

$$
\frac{\Delta_{\mathrm{mix}}}{m_{h}} \approx 1-\sqrt{\frac{1+\gamma^{2}\left(m_{s}^{2} / m_{h}^{2}\right)}{1+\gamma^{2}}} \approx \frac{1}{2} \frac{\gamma^{2}}{1+\gamma^{2}}\left(1-\frac{m_{s}^{2}}{m_{h}^{2}}\right) .
$$

For fixed $m_{s}$ and small $\gamma$ one gets the proportionality $\Delta_{\text {mix }} \propto \gamma^{2}$. From (6.1) we get the following values of the $\mathcal{B}_{\hat{h}_{i}}$ parameters:

$$
\mathcal{B}_{\hat{h}} \approx 1-\gamma \mathcal{A}_{s}, \quad \mathcal{B}_{\hat{H}} \approx 0, \quad \mathcal{B}_{\hat{s}} \approx \gamma+\mathcal{A}_{s} .
$$

Our $\mathcal{A}_{s}$ parameter can be expressed as

$$
\mathcal{A}_{s} \approx-\gamma \frac{1+c_{s}}{1+c_{h}}\left(\frac{m_{h}}{m_{s}}\right)^{2}
$$

where we introduced another convenient parameters

$$
c_{s} \equiv 1+\frac{\tilde{S}_{s \hat{H}}}{\tilde{S}_{s \hat{h}}}\left(\tan \beta-\frac{1}{\tan \beta}\right),
$$

\footnotetext{
${ }^{10}$ Such destructive interference was analyzed in some part of the parameter space of the scale-invariant NMSSM in ref. [42].

${ }^{11}$ Although, our approach holds for any $\tan \beta$, such terms are crucial in the analysis of the possible contribution to the SM-like Higgs scalar mass from the mixing with the light singlet-dominated scalar [24] when $\tan \beta$ is moderate or large.
} 


$$
c_{h} \equiv 1+\frac{\tilde{S}_{h \hat{H}}}{\tilde{S}_{h \hat{h}}}\left(\tan \beta-\frac{1}{\tan \beta}\right) .
$$

Without mixing with $\hat{H}$ the above quantities would be equal 1. In the limit of large $\tan \beta$ the $c_{s}\left(c_{h}\right)$ parameter measures the ratio of the couplings, normalized to SM values, of the $s(h)$ scalar to the $b$ quarks and to the $Z$ bosons. It is easier to make a light scalar $s$ compatible with the LEP bounds when $c_{s}$ is small [24], especially for $m_{s} \lesssim 85 \mathrm{GeV}$. We should note, however, that $c_{s}<1$ implies $c_{h}>1$ which in turn leads to suppressed branching ratios of $h$ decaying to gauge bosons, so $c_{h}$ is constrained by the LHC Higgs data.

Note that in contrary to $\mathcal{A}_{H}$ parameter (see (5.7)), $\mathcal{A}_{s}$ can have both signs depending mainly on the sign of $\gamma$. LEP and LHC constraints on $\gamma$, ranging from approximately 0.3 to 0.5 (corresponding to $m_{s}$ from $m_{h} / 2$ to about $100 \mathrm{GeV}$ ), imply that $\left|\mathcal{A}_{s}\right| \lesssim 1$ (the bound is saturated for $m_{s}$ around the LEP excess).

Because we assumed $\mathcal{B}_{\hat{H}} \approx 0$, the blind spot condition under consideration is of the form (3.17) and reads:

$$
\frac{\gamma+\mathcal{A}_{s}}{1-\gamma \mathcal{A}_{s}} \approx-\eta
$$

It is qualitatively different from the corresponding conditions in (4.9). The main reason is that the 1.h.s. of the above equation is not generically suppressed (in contrast to the cases considered in section 4.2). LEP and LHC constraints set upper bounds on $\left|\mathcal{B}_{\hat{s}} / \mathcal{B}_{\hat{h}}\right|$, nevertheless it can be as large as about $0.4(0.3)$ for $c_{s} \approx 1\left(c_{s} \approx 0\right)^{12}$ and therefore could be at least one order of magnitude larger than in the case with only $h$ exchange taken into account (see (4.9)).

The above blind spot condition may be rewritten in the form analogous to eq. (4.6):

$$
\frac{m_{\chi}}{\mu}-\sin 2 \beta \approx-\frac{\gamma+\mathcal{A}_{s}}{1-\gamma \mathcal{A}_{s}} \eta^{-1}\left(\frac{m_{\chi}}{\mu}-\sin 2 \beta\right) .
$$

There is one crucial modification as compared to $(4.6):^{13}$

$$
\gamma \longrightarrow \frac{\gamma+\mathcal{A}_{s}}{1-\gamma \mathcal{A}_{s}}
$$

Since $\left|\mathcal{B}_{\hat{s}} / \mathcal{B}_{\hat{h}}\right|$ does not have to be suppressed it is possible to have a blind spot for sizable values of $|\eta|$ independently of the sign of $m_{\chi} \mu$. This implies that a blind spot may occur for larger Higgsino-singlino mixing, even for $\lambda$ larger than $|\kappa|$. In particular, it is now possible to have a blind spot for a singlino-dominated LSP for large $\lambda$ and small $\tan \beta$ with sub-TeV $|\mu|$ for both signs of $m_{\chi} \mu$ without violating perturbativity up to the GUT scale. This is demonstrated in figure 5 . As can be seen for $\lambda=0.6$ and $\tan \beta=2$ the blind spots occur for $|\kappa| \lesssim$ 0.4 (which is necessary to avoid Landau poles below the GUT scale for this value of $\lambda$ ). This is in contrast to the case when $\sigma_{\mathrm{SI}}$ is dominated by only $h$ exchange, where for a singlinodominated LSP a blind spots with large $\lambda$ and small $\tan \beta$ were present only for $m_{\chi} \mu>0$.

In figure 6 an analogous plots to those presented for the heavy singlet case in figure 2 are shown. It can be seen that, if the singlet-dominated scalar is light, blind spots can

\footnotetext{
${ }^{12}$ These upper bounds are quite stable with respect to the change of $m_{s}$ between $m_{h} / 2$ and about $100 \mathrm{GeV}$.

${ }^{13}$ The first term in the r.h.s. of (4.6) is negligible in any case.
} 

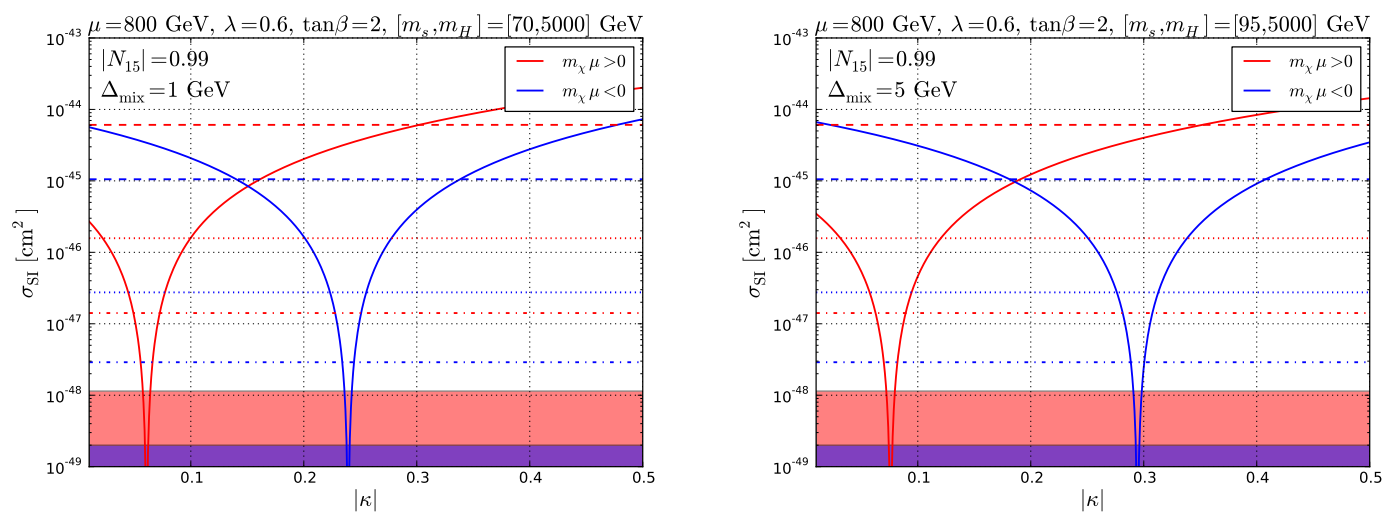

Figure 5. The LSP spin-independent cross-section (solid lines) for $\tan \beta=2$ as a function of $\kappa$ which sign is chosen two provide the same signs for both sides of (6.8). The horizontal lines show the experimental limits as in figure 1 . The colored regions depict the corresponding neutrino background levels. Plots for $\mu<0$ are very similar. The SD cross-section in the vicinity of blind spots is below the sensitivity of IceCube (independently of the assumed dominant annihilation channel).
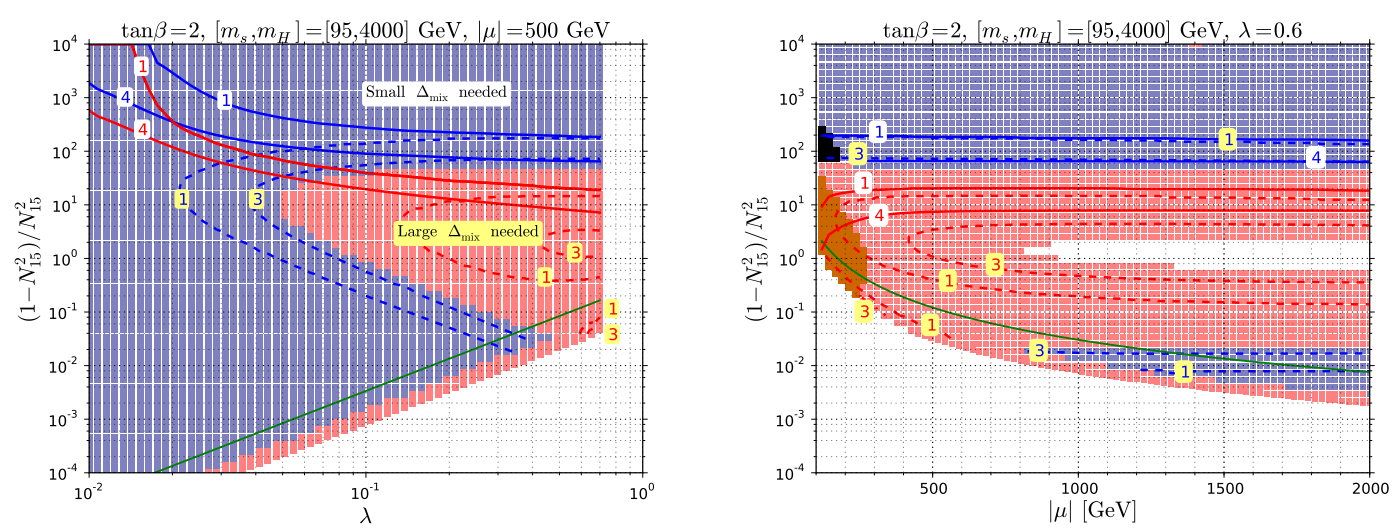

Figure 6. Left: regions of the plane $\left(\lambda,\left(1-N_{15}^{2}\right) / N_{15}^{2}\right)$ with the SI cross-section that can be below the neutrino background for $m_{\chi} \mu>0$ (red) and $m_{\chi} \mu<0$ (blue), while keeping $|\kappa| \leq 0.3$ and $\left|\Delta_{\text {mix }}\right|$ small enough to avoid the LEP and LHC constraints and $|\mu|=500 \mathrm{GeV}$. The solid contours correspond to maximal value of $\Delta_{\text {mix }}$ for which the SI scattering cross-section can be below the neutrino background - above these contours smaller $\Delta_{\text {mix }}$ is required for a blind spot to exist. The dashed contours correspond to minimal value of $\Delta_{\text {mix }}$ for which the SI scattering cross-section can be below the neutrino background - to the right of these contours larger $\Delta_{\text {mix }}$ is required for a blind spot to exist. Right: the same as in the left panel but as a function of $|\mu|$ for $\lambda=0.6$. Black (brown) region is excluded by the XENON100 constraints on the SD scattering cross-section [33] for $m_{\chi} \mu<0\left(m_{\chi} \mu>0\right)$. All points are consistent with the LHC and LEP Higgs data at $2 \sigma$. 
exist for large $\lambda$ and $\tan \beta=2$ without violating the perturbativity bounds for $m_{\chi} \mu>0$ for (almost) any composition of the LSP. The case of $m_{\chi} \mu<0$ is also less constrained. Nevertheless, if the LSP is not Higgsino-dominated, blind spots can exist for large $\lambda$ and $m_{\chi} \mu<0$ only for small range of $N_{15}$ (if $\kappa$ is kept in the perturbative regime). For low $\tan \beta$ the most interesting region is for large $\lambda$ so in the right panel of figure 6 we plot the regions where a blind spot can occur for fixed $\lambda=0.6$ as a function of $|\mu|$. It can be seen that for $m_{\chi} \mu<0$ a blind spot can occur for a singlino-dominated LSP if $|\mu| \gtrsim 800 \mathrm{GeV}$, and the range of possible values of $N_{15}$ grows with increasing $|\mu|$. For $m_{\chi} \mu>0$ almost any LSP composition allows for existence of a blind spot except for some region of a stronglymixed Higgsino-singlino LSP with $|\mu| \gtrsim 800 \mathrm{GeV}$ (in that region a blind spot cannot occur because $|\eta|$ is too large to satisfy the blind spot eq. (3.17) when the precision Higgs data, constraining the Higgs-singlet mixing, are taken into account).

The fact that the blind spots can now occur for large $\lambda$ and small $\tan \beta$ for much wider range of the LSP composition is not only due to the fact that the singlet-dominated scalar is light but also because of large Higgs-singlet mixing, hence also large $\Delta_{\text {mix }}$. This is demonstrated by dashed contours in figure 6 which correspond to minimal value of $\Delta_{\text {mix }}{ }^{14}$ for which the SI scattering cross-section may be below the neutrino background. It follows from the comparison of these contours with the plot in figure 2 (for heavy singlet) that $\Delta_{\text {mix }}$ above few $\mathrm{GeV}$ is required to significantly extend the range of the LSP composition for which a blind spot can occur when $\lambda$ is large.

It is also interesting to check what happens if one demands large $\Delta_{\operatorname{mix}}$ so that the Higgs scalar mass gets substantial enhancement from the Higgs-singlet mixing effects. In figure 6 we also present solid contours that correspond to maximal value of $\Delta_{\text {mix }}$ for which the SI scattering cross-section may be below the neutrino background. It can be seen that if one demands $\Delta_{\text {mix }}$ as small as $1 \mathrm{GeV}$ then for large $\lambda$ there are no blind spots for the LSP strongly dominated by the Higgsino component. This can be understood in the following way. For large $\Delta_{\text {mix }}$ and light singlet $\left|\mathcal{B}_{\hat{s}} / \mathcal{B}_{\hat{h}}\right|$ is no longer close to zero so in order for the blind spot to occur $|\eta|$ should not be close to zero. One can see from definition (3.12) that $|\eta| \sim\left|N_{15}\right|$ for the Higgsino-dominated case so a lower bound on $\Delta_{\text {mix }}$ sets a lower bound on the singlino component of the LSP. Noting that $\left|\mathcal{B}_{\hat{s}} / \mathcal{B}_{\hat{h}}\right|$ is in a good approximation proportional to $\sqrt{\left|\Delta_{\text {mix }}\right|}$, we conclude that a lower bound on $N_{15}^{2}$ scales proportionally to $\Delta_{\text {mix }}$. This is in agreement with the results in figure 6 .

Since in this case a SI cross-section blind spot can occur also for a highly-mixed Higgsino-singlino LSP one may expect to probe this region with SD direct detection experiments. Indeed, XENON100 limits exclude some part of the parameter space with SI cross-section blind spots for large $\lambda$ and small $|\mu|$ (black and brown points in figure 6). In this region of the parameter space the LSP annihilates dominantly to a light singlet-like scalar and a pseudoscalar, that typically decay to pairs of bottom quarks so the IceCube limits are not expected to be stronger than the XENON100 ones.

\footnotetext{
${ }^{14}$ Note that the results in figure 6 come from a scan of four parameters: $N_{15}, \lambda, \Delta_{\text {mix }}$ and $\kappa$. Therefore, for a given point in the $N_{15}-\lambda$ plane there might be several solutions with the SI scattering cross-section below the neutrino background with different values of $\Delta_{\text {mix }}$.
} 
We should emphasize that the effect of large Higgs-singlet mixing has particularly important implications for models with $\mu^{\prime}=0$ (i.e. with vanishing quadratic term in $f(S)$ ), including the $Z_{3}$-invariant NMSSM, because in those models the LSP composition is related to the ratio $\kappa / \lambda$. Namely, the LSP is singlino-dominated if $\lambda>2|\kappa|$. This implies that for large $\lambda$, the LSP is typically singlino-dominated and can be highly mixed Higgsinosinglino only if $|\kappa|$ is close to the upper bound from the requirement of perturbativity up to the GUT scale. In consequence, in this class of NMSSM models with large $\lambda$ and small $\tan \beta$ a blind spot may occur only for $2 \kappa / \lambda \approx m_{\chi} / \mu \approx \sin (2 \beta)$ if the Higgs-singlet mixing is small. On the other hand, for large Higgs-singlet mixing a blind spot can occur for much wider range of $\kappa / \lambda$ (corresponding to different LSP compositions) for $m_{\chi} \mu>0$, while for $m_{\chi} \mu<0$ existence of a blind spot may be possible provided that $|\mu|$ is large enough.

\subsection{Large $\tan \beta$ region}

In models with large $\tan \beta$, couplings of $s$ and $h$ scalars to $b$ quarks may significantly deviate from the couplings to the massive gauge bosons which has important consequences for the SI scattering cross-section. From our perspective the most interesting situation takes place when $\Delta_{\text {mix }}$, being now positive, is large. As stated above, for $m_{s} \lesssim 85 \mathrm{GeV}$ small $\left|c_{s}\right|$ and hence large $\tan \beta$ and small $\lambda$ are preferred [24]. For definiteness, let us consider $\tan \beta=10, \lambda=0.1$ and two representative values of $m_{s}, 70$ and $95 \mathrm{GeV}$, for which the LEP bounds are, respectively, quite severe and rather mild. In figure 7 we present the points (for a few values of $c_{s}$ ) for which $\sigma_{\mathrm{SI}}$ is smaller than the neutrino background for two signs of $m_{\chi} \mu$. The most apparent difference between $c_{s}>1$ and $c_{s}<1$ is that in the first case there are no points with a Higgsino-dominated LSP, whereas in the second one there is a negative correlation between Higgsino admixture and $\Delta_{\text {mix }}$ (for $N_{15}^{2} \lesssim 0.1$ ). In order to explain this behavior we rewrite the blind spot condition (6.8) in the form adequate for the Higgsino-dominated limit i.e. for $\left|m_{\chi} / \mu\right| \rightarrow 1$. The result reads:

$$
\frac{\gamma+\mathcal{A}_{s}}{1-\gamma \mathcal{A}_{s}} \approx \operatorname{sgn}(\mu)\left|N_{15}\right| \sqrt{2\left(1-\operatorname{sgn}\left(m_{\chi} \mu\right) \sin 2 \beta\right)} .
$$

For specific values of $c_{s}$ and $m_{s}$ (chosen in our example) the 1.h.s. of the above equation is proportional to $\gamma$ (with a negative coefficient ${ }^{15}$ ) and thus to $\sqrt{\Delta_{\text {mix }}}($ see $(6.2)$ ) - this explains why there is a correlation between $\Delta_{\text {mix }}$ and $\left|N_{15}\right|$. To understand why for $c_{s}>1$ $\left(c_{s}<1\right)$ there are (no) points which fulfill $(6.10)$ we should notice (see eqs $\left.(2.8),(2.9)\right)$ that for $\tan \beta \gg 1$ we have $\operatorname{sgn}\left(1-c_{s}\right)=\operatorname{sgn}(\Lambda \gamma)=\operatorname{sgn}(\mu \gamma)$ - the second equality holds because a partial cancellation between the two terms in $M_{h \hat{s}}^{2}$ is needed. ${ }^{16}$ This is exactly what we wanted to show: for $c_{s}<1$ the l.h.s. of (6.10) has the sign equal to $-\operatorname{sgn}(\mu)$ thus the equality cannot hold (and inversely for $c_{s}>1$ ). It can be shown (using relations (2.12) and (2.13)), that the above conclusions hold also for some part of a highly mixed LSP parameter space

\footnotetext{
${ }^{15}$ It can be easily seen if we notice that $\mathcal{A}_{s}=-\gamma$ const, where const $=\frac{1+c_{s}}{1+c_{h}}\left(\frac{m_{h}}{m_{s}}\right)^{2}>1$. Moreover $\left|\gamma \mathcal{A}_{s}\right| \ll 1$ and hence the denominator in the l.h.s. of (6.10) is roughly 1 .

${ }^{16}$ This happens in our example in figure 7 because for $|\mu|=500 \mathrm{GeV}$ and $\lambda=0.1$ we have $\left|M_{h \hat{s}}^{2}\right| \sim$ $\mathcal{O}(100 \mathrm{GeV})$, which is of order $m_{h}$ and $m_{s}$. The situation for smaller $|\mu|$ is not much different.
} 

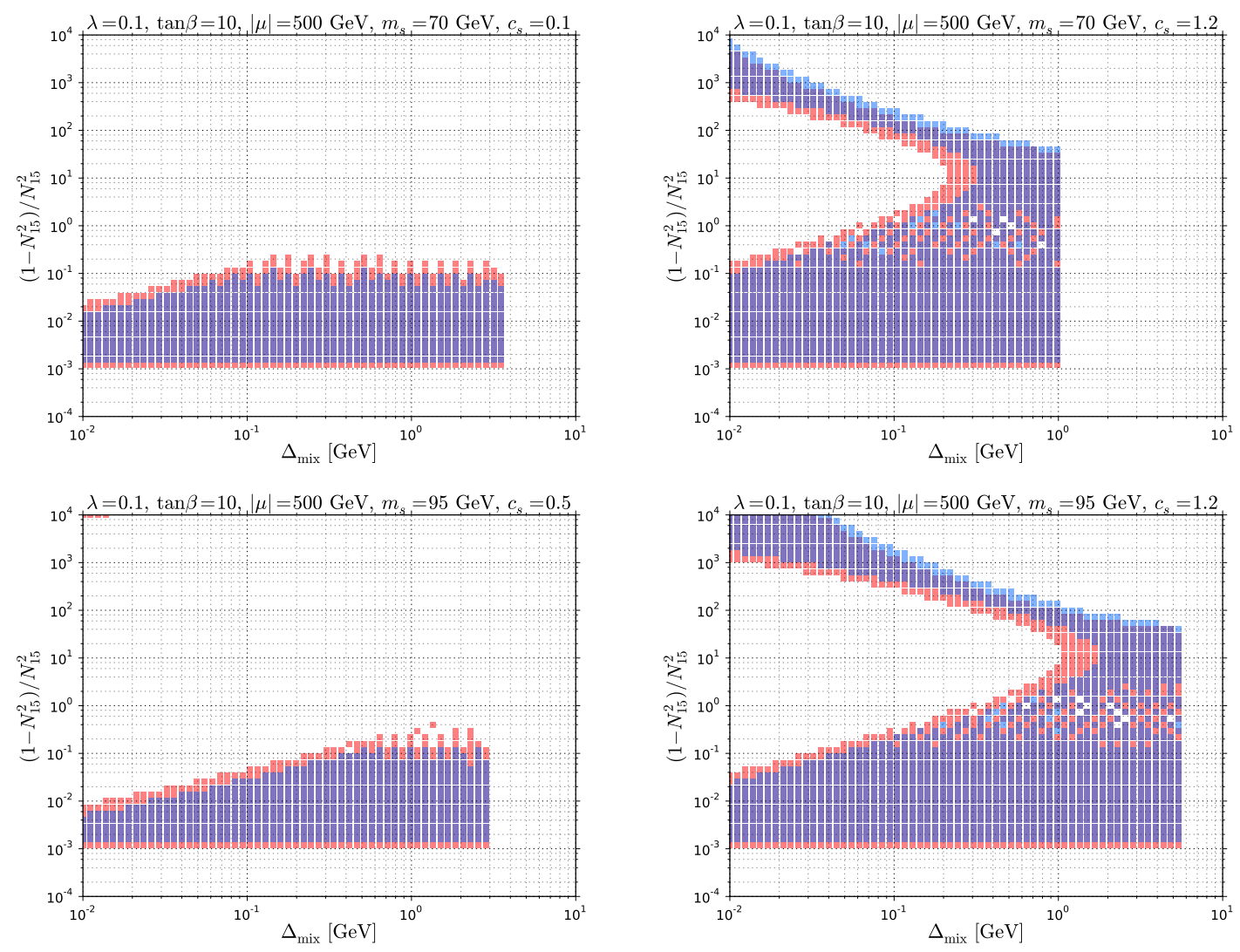

Figure 7. Regions of the plane $\left(\Delta_{\text {mix }},\left(1-N_{15}^{2}\right) / N_{15}^{2}\right)$ with $\sigma_{\mathrm{SI}}$ smaller than the neutrino background [6] for $m_{\chi} \mu>0$ (red) and $m_{\chi} \mu<0$ (blue), while keeping $|\kappa| \leq 0.6$. Upper (lower) plots correspond to $m_{s}=70(95) \mathrm{GeV}$ whereas the left (right) to $c_{s}$ smaller (larger) than 1.

when $|\kappa / \lambda|$ is smaller than $\left|\frac{N_{13}}{N_{15}} \frac{N_{14}}{N_{15}}\right|$ i.e. with unsuppressed $|\eta|$ in eq. (6.8). For a singlinodominated LSP we can always choose the sign and value of $\kappa$ to fulfill relation (6.8).

Let us finally comment on the fact that for large $\tan \beta$ the $H$ exchange might be relevant if $H$ is light enough. The presence of relatively light $H$ usually results in stronger constraints on the parameter space, especially for large values of $\lambda$. This is because in this region of the parameter space $\left|M_{\hat{H} \hat{s}}^{2}\right|$ is well approximated by $\lambda v \mu \tan \beta$ so it is typically larger than the diagonal entries of the Higgs mass matrix, unless $\lambda$ is small. As a result, large values of $\lambda$ lead to tachyons, or at least the mixing effects that are too large to accommodate the LEP and/or LHC Higgs data.

\section{Summary}

We have investigated blind spots for spin-independent scattering cross-section for the Higgsino-singlino LSP in the NMSSM. If mixing between the (SM-like) Higgs scalar and other scalars is negligible, a blind spot can occur only if the ratio $m_{\chi} / \mu$ is positive and has value close to $\sin 2 \beta$. Then, blind spots exist only for singlino-dominated LSPs (unless 
$\tan \beta$ is very close to 1 ) with the amount of the Higgsino component determined by $\tan \beta$. This changes a lot when mixing with the singlet scalar is taken into account.

If the singlet-dominated scalar is heavier than the Higgs scalar, the Higgs-singlet mixing has to be quite small to avoid large negative correction to the Higgs scalar mass. But even for such small mixing new classes of blind spots appear. Blind spots for Higgsino-dominated LSPs become possible and the ratio $m_{\chi} / \mu$ may be also negative. The LSP composition is no longer so strongly related to $\tan \beta$, especially for smaller values of $\lambda$. However, in most cases the LSP must be highly dominated either by Higgsino or by singlino. A blind spot for a highly mixed Higgsino-singlino LSP is possible only for small values of $\lambda$ and $\tan \beta$ and positive $m_{\chi} / \mu$. In addition, in the most often explored part of NMSSM parameter space with large (but perturbative) $\lambda$ and small $\tan \beta$, a blind spot for a singlino-dominated LSP can occur only if $m_{\chi} \mu>0$ and eq. (4.2) is approximately satisfied.

If the singlet-dominated scalar is lighter than the Higgs scalar, large Higgs-singlet mixing is welcome because the contribution from such mixing to the Higgs scalar mass is positive. For small $\tan \beta$, the LEP and LHC constraints allow for sizable mixing leading to the correction to the Higgs scalar mass $\Delta_{\text {mix }} \sim 5 \mathrm{GeV}$ for the singlet mass in the range of about $85 \div 105 \mathrm{GeV}$. For such big $\Delta_{\text {mix }}$, a blind spot for large $\lambda$ and $\tan \beta \sim 2$ may occur also for highly mixed Higgsino-singlino LSP if $m_{\chi} \mu>0$, which would not be possible otherwise. It should be noted, however, that not always large $\Delta_{\text {mix }}$ is beneficial for a blind spot occurrence. For example, for an LSP strongly dominated by the Higgsino a blind spot may occur only if $\Delta_{\text {mix }}$ is small.

For light singlet scalar and big $\Delta_{\text {mix }}$ the region of moderate and large $\tan \beta$ is also interesting. In such a case the singlet coupling to bottom quarks may be significantly different than the one to gauge bosons. If the $s b \bar{b}$ coupling is suppressed, relatively large $\Delta_{\text {mix }}$ is allowed by LEP also for $m_{s}<85 \mathrm{GeV}$. We found that for suppressed $s b \bar{b}$ coupling a blind spot may occur only for a singlino-dominated LSP. On the other hand, if the $s b \bar{b}$ coupling is enhanced a blind spot can exist for any composition of the LSP and for both signs of $m_{\chi} \mu$.

For large $\tan \beta$ one more class of blind spots may exist if the heavier scalar doublet $H$ is light enough to mediate the LSP-nucleon interaction in a substantial way and the singlet-dominated scalar is rather heavy. In such a case, positive $m_{\chi} \mu$ is again preferred, allowing for blind spots for the LSP composition much less restricted than in the case with very heavy $H$. If the Higgs-singlet mixing is present, $m_{\chi} \mu<0$ is also possible but in this case the influence of a relatively light $H$ on possible blind spots is quite marginal. In addition, smaller values of $m_{H}$ result in stronger upper bounds on the coupling $\lambda$.

There are several avenues for future studies where the results obtained in this paper can be used. For instance, it will be crucial to investigate how one can probe neutralino LSP with SI scattering cross-section below the neutrino background. Some possible ways to constrain blind spots may be to use the direct and indirect detection experiments sensitive to the SD cross-sections or dedicated collider searches which in the context of MSSM turn out to be complementary to direct dark matter searches, see e.g. [28, 43, 44] for some recent work on this topic. Some studies of the LHC sensitivity to Higgsino-singlino sector has already been done [45] but more effort in this direction is welcome. It will be also interesting to investigate whether the blind spots identified in this paper can exist in more 
constrained versions of NMSSM and in which scenarios it is possible to explain the observed abundance of dark matter assuming thermal history of the Universe. We plan to investigate these issues in the future.

\section{Acknowledgments}

This work was partially supported by Polish National Science Centre under research grants DEC-2012/05/B/ST2/02597, DEC-2014/15/B/ST2/02157 and DEC2012/04/A/ST2/00099. MB acknowledges support from the Polish Ministry of Science and Higher Education (decision no. 1266/MOB/IV/2015/0). MB thanks the Galileo Galilei Institute for Theoretical Physics and INFN for hospitality and partial support during the completion of this work.

\section{A Useful formulae}

The parameter $\eta$ defined in (3.12) may be expressed in terms of other parameters of the NMSSM model. With the help of eqs. (2.12) and (2.13) one can write it as the following function of three dimensionless ratios: $(\lambda v) / \mu, \kappa / \lambda$ and $m_{\chi} / \mu$ :

$$
\eta=\frac{\frac{\lambda v}{\mu}\left(1-\left(\frac{m_{\chi}}{\mu}\right)^{2}\right)\left(\frac{m_{\chi}}{\mu}-\sin 2 \beta\right)}{\left(\frac{\lambda v}{\mu}\right)^{2}\left[\left(1+\left(\frac{m_{\chi}}{\mu}\right)^{2}\right) \frac{\sin 2 \beta}{2}-\frac{m_{\chi}}{\mu}\right]-\frac{\kappa}{\lambda}\left(1-\left(\frac{m_{\chi}}{\mu}\right)^{2}\right)^{2}} .
$$

Equation (2.14) may be used to eliminate $(\lambda v) / \mu$ in favor of the ratio $\left(1-N_{15}^{2}\right) / N_{15}^{2}$ characterizing the LSP composition. Then, one obtains another expression for $\eta$ :

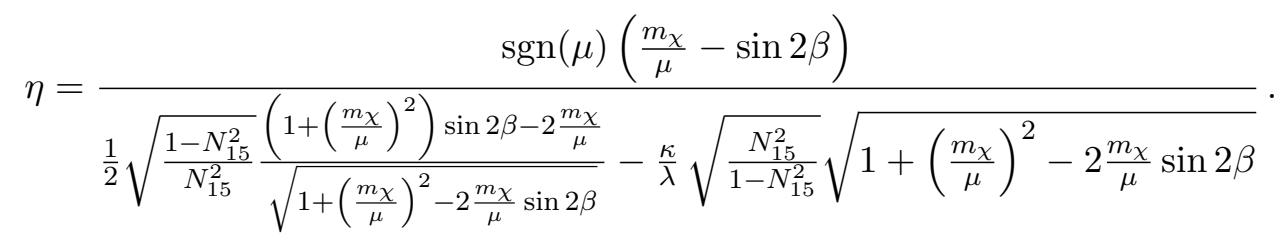

It will be helpful to consider a few limits of this parameter. Let us start with the situation when one of the terms in the denominator dominates over the other one. The first (second) term in the denominator may be neglected if $\left|\frac{\kappa}{\lambda}\right|$ is much bigger (smaller) than $\frac{1-N_{15}^{2}}{2 N_{15}^{2}}\left|\frac{\left(m_{\chi} / \mu+\mu / m_{\chi}\right) \sin 2 \beta-2}{\left(m_{\chi} / \mu+\mu / m_{\chi}\right)-2 \sin 2 \beta}\right|$. The second factor in the last expression is always smaller than 1 and approaches 1 in the limit $\left|m_{\chi} / \mu\right| \rightarrow 1$ i.e. for a strongly Higgsino-dominated LSP. It may be very small if $m_{\chi} \mu>0$ and $\sin 2 \beta \approx 2 /\left(m_{\chi} / \mu+\mu / m_{\chi}\right)$.

If $\left|\frac{\kappa}{\lambda}\right| \gg \frac{1-N_{15}^{2}}{2 N_{15}^{2}}\left|\frac{\left(m_{\chi} / \mu+\mu / m_{\chi}\right) \sin 2 \beta-2}{\left(m_{\chi} / \mu+\mu / m_{\chi}\right)-2 \sin 2 \beta}\right|>\frac{1-N_{15}^{2}}{2 N_{15}^{2}}$ i.e. we are considering a singlinodominated LSP and/or $|\kappa|$ much bigger than $\lambda$ (for a not strongly Higgsino-dominated LSP), the parameter $\eta$ is approximately given by:

$$
\eta \approx-\operatorname{sgn}(\mu) \frac{\lambda}{\kappa} \sqrt{\frac{1-N_{15}^{2}}{N_{15}^{2}}} \frac{\frac{m_{\chi}}{\mu}-\sin 2 \beta}{\sqrt{1+\left(\frac{m_{\chi}}{\mu}\right)^{2}-2 \frac{m_{\chi}}{\mu} \sin 2 \beta}} .
$$


If $\left|\frac{\kappa}{\lambda}\right| \ll \frac{1-N_{15}^{2}}{2 N_{15}^{2}}\left|\frac{\left(m_{\chi} / \mu+\mu / m_{\chi}\right) \sin 2 \beta-2}{\left(m_{\chi} / \mu+\mu / m_{\chi}\right)-2 \sin 2 \beta}\right|$ i.e. for a Higgsino-dominated LSP and/or $|\kappa|$ is much smaller than $\lambda$ (for a not strongly singlino-dominated LSP) we get:

$$
\eta \approx \operatorname{sgn}(\mu) \sqrt{\frac{N_{15}^{2}}{1-N_{15}^{2}}} \frac{2\left(\frac{m_{\chi}}{\mu}-\sin 2 \beta\right) \sqrt{1+\left(\frac{m_{\chi}}{\mu}\right)^{2}-2 \frac{m_{\chi}}{\mu} \sin 2 \beta}}{\left(1+\left(\frac{m_{\chi}}{\mu}\right)^{2}\right) \sin 2 \beta-2 \frac{m_{\chi}}{\mu}} .
$$

In the case of a strongly Higgsino-dominated LSP, using $N_{15}^{2} \ll 1$ and $m_{\chi}^{2} \approx \mu^{2}$, the above equality may be further approximated as:

$$
\eta \approx-\operatorname{sgn}(\mu)\left|N_{15}\right| \sqrt{2\left(1-\operatorname{sgn}\left(m_{\chi} \mu\right) \sin 2 \beta\right)} .
$$

Above there are several forms and limits of the parameter $\eta$ defined in eq. (3.12). With the help of eqs. (2.12)-(2.14) one may rewrite also the whole amplitude (3.9) as:

$$
\begin{aligned}
f^{(N)} \approx & \frac{\lambda}{\sqrt{2}} \frac{\alpha_{h N N}}{m_{h}^{2}} \tilde{S}_{h \hat{h}} \sqrt{\frac{N_{15}^{2}\left(1-N_{15}^{2}\right)}{1+\left(\frac{m_{\chi}}{\mu}\right)^{2}-2 \frac{m_{\chi}}{\mu} \sin 2 \beta}}\left\{\mathcal{B}_{\hat{h}}\left[\frac{m_{\chi}}{\mu}-\sin 2 \beta\right]+\mathcal{B}_{\hat{H}} \cos 2 \beta\right. \\
& \left.+\mathcal{B}_{\hat{s}}\left[\frac{\frac{\lambda v}{\mu}}{1-\left(\frac{m_{\chi}}{\mu}\right)^{2}}\left(\left(1+\left(\frac{m_{\chi}}{\mu}\right)^{2}\right) \frac{\sin 2 \beta}{2}-\frac{m_{\chi}}{\mu}\right)-\frac{\kappa}{\lambda} \frac{1-\left(\frac{m_{\chi}}{\mu}\right)^{2}}{\frac{\lambda v}{\mu}}\right]\right\}
\end{aligned}
$$

This formula is not very convenient in the case of a strongly Higgsino-dominated LSP. In such limit $N_{15} \rightarrow 0$ but one of the terms in the curly bracket diverges as $m_{\chi}^{2} \rightarrow \mu^{2}$. Thus, for a strongly Higgsino-dominated LSP it is better to rewrite eq. (A.6) in the following form:

$$
\begin{aligned}
f^{(N)} \approx & \frac{\lambda}{\sqrt{2}} \frac{\alpha_{h N N}}{m_{h}^{2}} \frac{\tilde{S}_{h \hat{h}}\left(1-N_{15}^{2}\right)}{1+\left(\frac{m_{\chi}}{\mu}\right)^{2}-2 \frac{m_{\chi}}{\mu} \sin 2 \beta}\left\{\mathcal{B}_{\hat{h}} \frac{1-\left(\frac{m_{\chi}}{\mu}\right)^{2}}{\frac{\lambda v}{\mu}}\left[\frac{m_{\chi}}{\mu}-\sin 2 \beta\right]\right. \\
& \left.+\mathcal{B}_{\hat{H}} \frac{1-\left(\frac{m_{\chi}}{\mu}\right)^{2}}{\frac{\lambda v}{\mu}} \cos 2 \beta+\mathcal{B}_{\hat{s}}\left[\left(1+\left(\frac{m_{\chi}}{\mu}\right)^{2}\right) \frac{\sin 2 \beta}{2}-\frac{m_{\chi}}{\mu}-\frac{\kappa}{\lambda}\left(\frac{1-\left(\frac{m_{\chi}}{\mu}\right)^{2}}{\frac{\lambda v}{\mu}}\right)^{2}\right]\right\} .
\end{aligned}
$$

Comments on the spin-dependent scattering cross-section. The only contribution at the tree-level to the spin-dependent scattering cross-section in our case comes from the $t$-channel $Z$ exchange, so depends only on the Higgsino contribution to the LSP and reads:

$$
\sigma_{\mathrm{SD}}^{(N)}=C^{(N)} \cdot 10^{-38} \mathrm{~cm}^{2}\left(N_{13}^{2}-N_{14}^{2}\right)^{2},
$$

where $C^{(p)} \approx 4, C^{(n)} \approx 3.1[46,47]$. Combining eqs. (2.12), (2.13) and (2.14) we can write:

$$
N_{13}^{2}-N_{14}^{2}=\frac{\left[1-\left(m_{\chi} / \mu\right)^{2}\right]\left(1-N_{15}^{2}\right) \cos 2 \beta}{1+\left(m_{\chi} / \mu\right)^{2}-2\left(m_{\chi} / \mu\right) \sin 2 \beta} .
$$


We can see immediately that the cross-section disappear in the limit of $\tan \beta=1$ or a pure singlino/Higgsino LSP. Using eq. (2.14) we may rewrite the last formula in the form

$$
N_{13}^{2}-N_{14}^{2}=\frac{\left[1-\left(m_{\chi} / \mu\right)^{2}\right] \cos 2 \beta}{1+\left(m_{\chi} / \mu\right)^{2}-2\left(m_{\chi} / \mu\right) \sin 2 \beta+\left[1-\left(m_{\chi} / \mu\right)^{2}\right]^{2}(\mu / \lambda v)^{2}}
$$

showing the explicit dependence of the LSP- $Z$ coupling on $\lambda$ (there is also an implicit dependence via the LSP mass $m_{\chi}$ ).

Open Access. This article is distributed under the terms of the Creative Commons Attribution License (CC-BY 4.0), which permits any use, distribution and reproduction in any medium, provided the original author(s) and source are credited.

\section{References}

[1] ATLAS collaboration, Observation of a new particle in the search for the standard model Higgs boson with the ATLAS detector at the LHC, Phys. Lett. B 716 (2012) 1 [arXiv: 1207.7214] [INSPIRE].

[2] CMS collaboration, Observation of a new boson at a mass of $125 \mathrm{GeV}$ with the CMS experiment at the LHC, Phys. Lett. B 716 (2012) 30 [arXiv:1207.7235] [INSPIRE].

[3] LUX collaboration, D.S. Akerib et al., First results from the LUX dark matter experiment at the Sanford Underground Research Facility, Phys. Rev. Lett. 112 (2014) 091303 [arXiv: 1310.8214] [INSPIRE].

[4] XENON collaboration, E. Aprile et al., Physics reach of the XENON1T dark matter experiment, submitted to JCAP (2015) [arXiv:1512.07501] [INSPIRE].

[5] D.C. Malling et al., After LUX: the LZ program, arXiv:1110.0103 [INSPIRE].

[6] J. Billard, L. Strigari and E. Figueroa-Feliciano, Implication of neutrino backgrounds on the reach of next generation dark matter direct detection experiments, Phys. Rev. D 89 (2014) 023524 [arXiv: 1307.5458] [INSPIRE].

[7] Planck collaboration, P.A.R. Ade et al., Planck 2015 results. XIII. Cosmological parameters, arXiv: 1502.01589 [INSPIRE].

[8] C. Cheung, L.J. Hall, D. Pinner and J.T. Ruderman, Prospects and blind spots for neutralino dark matter, JHEP 05 (2013) 100 [arXiv:1211.4873] [INSPIRE].

[9] P. Huang and C.E.M. Wagner, Blind spots for neutralino dark matter in the MSSM with an intermediate $m_{A}$, Phys. Rev. D 90 (2014) 015018 [arXiv:1404.0392] [INSPIRE].

[10] U. Ellwanger, C. Hugonie and A.M. Teixeira, The next-to-minimal supersymmetric standard model, Phys. Rept. 496 (2010) 1 [arXiv:0910.1785] [INSPIRE].

[11] B.R. Greene and P.J. Miron, Supersymmetric cosmology with a gauge singlet, Phys. Lett. B 168 (1986) 226 [INSPIRE].

[12] R. Flores, K.A. Olive and D. Thomas, A new dark matter candidate in the minimal extension of the supersymmetric standard model, Phys. Lett. B 245 (1990) 509 [INSPIRE].

[13] G. Bélanger, F. Boudjema, C. Hugonie, A. Pukhov and A. Semenov, Relic density of dark matter in the NMSSM, JCAP 09 (2005) 001 [hep-ph/0505142] [INSPIRE]. 
[14] D.G. Cerdeno, C. Hugonie, D.E. Lopez-Fogliani, C. Muñoz and A.M. Teixeira, Theoretical predictions for the direct detection of neutralino dark matter in the NMSSM, JHEP 12 (2004) 048 [hep-ph/0408102] [INSPIRE].

[15] D.G. Cerdeno, E. Gabrielli, D.E. Lopez-Fogliani, C. Muñoz and A.M. Teixeira, Phenomenological viability of neutralino dark matter in the NMSSM, JCAP 06 (2007) 008 [hep-ph/0701271] [INSPIRE].

[16] V. Barger, P. Langacker, I. Lewis, M. McCaskey, G. Shaughnessy and B. Yencho, Recoil detection of the lightest neutralino in MSSM singlet extensions, Phys. Rev. D 75 (2007) 115002 [hep-ph/0702036] [inSPIRE].

[17] D. Das and U. Ellwanger, Light dark matter in the NMSSM: upper bounds on direct detection cross sections, JHEP 09 (2010) 085 [arXiv: 1007.1151] [INSPIRE].

[18] J. Kozaczuk and S. Profumo, Light NMSSM neutralino dark matter in the wake of CDMS II and a 126 GeV Higgs boson, Phys. Rev. D 89 (2014) 095012 [arXiv: 1308.5705] [InSPIRE].

[19] J. Cao, C. Han, L. Wu, P. Wu and J.M. Yang, A light SUSY dark matter after CDMS-II, LUX and LHC Higgs data, JHEP 05 (2014) 056 [arXiv: 1311.0678] [INSPIRE].

[20] T. Han, Z. Liu and S. Su, Light neutralino dark matter: direct/indirect detection and collider searches, JHEP 08 (2014) 093 [arXiv: 1406.1181] [INSPIRE].

[21] R. Enberg, S. Munir, C. Pérez de los Heros and D. Werder, Prospects for higgsino-singlino dark matter detection at IceCube and PINGU, arXiv:1506.05714 [INSPIRE].

[22] C. Cheung and D. Sanford, Simplified models of mixed dark matter, JCAP 02 (2014) 011 [arXiv: 1311.5896] [INSPIRE].

[23] L. Calibbi, A. Mariotti and P. Tziveloglou, Singlet-doublet model: dark matter searches and LHC constraints, JHEP 10 (2015) 116 [arXiv:1505.03867] [INSPIRE].

[24] M. Badziak, M. Olechowski and S. Pokorski, New regions in the NMSSM with a $125 \mathrm{GeV}$ Higgs, JHEP 06 (2013) 043 [arXiv: 1304.5437] [INSPIRE].

[25] DelPhi, OPAL, ALEPH, LEP Working Group for Higgs Boson Searches and L3 collaborations, S. Schael et al., Search for neutral MSSM Higgs bosons at LEP, Eur. Phys. J. C 47 (2006) 547 [hep-ex/0602042] [INSPIRE].

[26] LEP Higgs Working Group For Higgs boson SEARChes collaboration, Flavor independent search for hadronically decaying neutral Higgs bosons at LEP, hep-ex/0107034 [INSPIRE].

[27] U. Ellwanger and C. Hugonie, The semi-constrained NMSSM satisfying bounds from the LHC, LUX and Planck, JHEP 08 (2014) 046 [arXiv: 1405.6647] [INSPIRE].

[28] M. Badziak, A. Delgado, M. Olechowski, S. Pokorski and K. Sakurai, Detecting underabundant neutralinos, JHEP 11 (2015) 053 [arXiv: 1506.07177] [INSPIRE].

[29] G. Jungman, M. Kamionkowski and K. Griest, Supersymmetric dark matter, Phys. Rept. 267 (1996) 195 [hep-ph/9506380] [INSPIRE].

[30] U. Ellwanger, J.F. Gunion and C. Hugonie, NMHDECAY: a fortran code for the Higgs masses, couplings and decay widths in the NMSSM, JHEP 02 (2005) 066 [hep-ph/0406215] [INSPIRE]. 
[31] U. Ellwanger and C. Hugonie, NMHDECAY 2.0: an updated program for sparticle masses, Higgs masses, couplings and decay widths in the NMSSM, Comput. Phys. Commun. 175 (2006) 290 [hep-ph/0508022] [INSPIRE].

[32] G. Bélanger, F. Boudjema, A. Pukhov and A. Semenov, MicrOMEGAs 3 : a program for calculating dark matter observables, Comput. Phys. Commun. 185 (2014) 960 [arXiv: 1305.0237] [INSPIRE].

[33] XENON100 collaboration, E. Aprile et al., Limits on spin-dependent WIMP-nucleon cross sections from 225 live days of XENON100 data, Phys. Rev. Lett. 111 (2013) 021301 [arXiv: 1301.6620] [INSPIRE].

[34] ICECube collaboration, M.G. Aartsen et al., Improved limits on dark matter annihilation in the sun with the 79-string IceCube detector and implications for supersymmetry, arXiv: 1601.00653 [INSPIRE].

[35] R. Barbieri, L.J. Hall, Y. Nomura and V.S. Rychkov, Supersymmetry without a light Higgs boson, Phys. Rev. D 75 (2007) 035007 [hep-ph/0607332] [INSPIRE].

[36] L.J. Hall, D. Pinner and J.T. Ruderman, A natural SUSY Higgs near 126 GeV, JHEP 04 (2012) 131 [arXiv:1112.2703] [INSPIRE].

[37] J. Hisano, K. Ishiwata, N. Nagata and T. Takesako, Direct detection of electroweak-interacting dark matter, JHEP 07 (2011) 005 [arXiv:1104.0228] [INSPIRE].

[38] J. Hisano, K. Ishiwata and N. Nagata, QCD effects on direct detection of wino dark matter, JHEP 06 (2015) 097 [arXiv: 1504.00915] [INSPIRE].

[39] R.J. Hill and M.P. Solon, Universal behavior in the scattering of heavy, weakly interacting dark matter on nuclear targets, Phys. Lett. B 707 (2012) 539 [arXiv:1111.0016] [INSPIRE].

[40] ATLAS collaboration, Search for neutral Higgs bosons of the minimal supersymmetric standard model in pp collisions at $\sqrt{s}=8 \mathrm{TeV}$ with the ATLAS detector, JHEP 11 (2014) 056 [arXiv: 1409.6064] [INSPIRE].

[41] CMS collaboration, Search for neutral MSSM Higgs bosons decaying to a pair of tau leptons in pp collisions, JHEP 10 (2014) 160 [arXiv:1408.3316] [INSPIRE].

[42] J. Cao, L. Shang, P. Wu, J.M. Yang and Y. Zhang, Interpreting the galactic center gamma-ray excess in the NMSSM, JHEP 10 (2015) 030 [arXiv:1506.06471] [INSPIRE].

[43] D. Barducci, A. Belyaev, A.K.M. Bharucha, W. Porod and V. Sanz, Uncovering natural supersymmetry via the interplay between the LHC and direct dark matter detection, JHEP 07 (2015) 066 [arXiv: 1504.02472] [INSPIRE].

[44] J. Cao, Y. He, L. Shang, W. Su and Y. Zhang, Testing the light dark matter scenario of the MSSM at the LHC, arXiv:1511.05386 [INSPIRE].

[45] U. Ellwanger, Testing the higgsino-singlino sector of the NMSSM with trileptons at the LHC, JHEP 11 (2013) 108 [arXiv:1309.1665] [INSPIRE].

[46] G. Chalons, M.J. Dolan and C. McCabe, Neutralino dark matter and the Fermi gamma-ray lines, JCAP 02 (2013) 016 [arXiv:1211.5154] [INSPIRE].

[47] G. Bélanger, F. Boudjema, A. Pukhov and A. Semenov, Dark matter direct detection rate in a generic model with MicrOMEGAs 2.2, Comput. Phys. Commun. 180 (2009) 747 [arXiv:0803.2360] [INSPIRE]. 